\title{
A NEW APPROACH TO THE FEASIBILITY PUMP IN MIXED INTEGER PROGRAMMING*
}

\author{
N. L. BOLAND ${ }^{\dagger}$, A. C. EBERHARD ${ }^{\ddagger}$, F. ENGINEER ${ }^{\dagger}$, AND A. TSOUKALAS $^{\ddagger}$
}

\begin{abstract}
The feasibility pump is a recent, highly successful heuristic for general mixed integer linear programming problems. We show that the feasibility pump heuristic can be interpreted as a discrete version of the proximal point algorithm. In doing so, we extend and generalize some of the fundamental results in this area to provide new supporting theory. We show that feasibility pump algorithms implicitly minimize a weighted combination of the objective and a term which penalizes lack of integrality. This function has many local minima, some of which correspond to feasible integral solutions; the feasibility pump's use of random restarts can be viewed as seeking to escape these local minima when they are not feasible integral solutions. This interpretation suggests alternative ways of incorporating restarts, one of which is the application of cutting planes. Numerical experiments with cutting planes show encouraging results on standard test libraries.
\end{abstract}

Key words. feasibility pump, proximal points, Moreau regularization, integer programming, cutting planes

AMS subject classifications. 90C10, 90C06, 90C59

DOI. $10.1137 / 110823596$

1. Introduction. In spite of the continuous improvement of both commercial and open source solvers, numerous mixed integer programming (MIP) problems of practical importance remain intractable. In practice, where rigorous algorithms fail, heuristics often succeed to provide feasible solutions of good quality. Apart from their self-evident value, good feasible solutions are also useful in speeding up the search of branch and cut algorithms. General purpose heuristics include $[2,3,4,13,14,15,16$, $19,20,21,22,23]$. We direct the reader to [6] for a recent survey.

A heuristic that has attracted significant interest in recent years is the feasibility pump (FP) [17]. The FP starts from the linear program (LP) optimum and computes two trajectories of points, one integer and the other LP feasible, by iteratively applying rounding and projection operations. Every LP feasible point is rounded to an integer point and every integer point is projected back onto the LP feasible region using the $l_{1}$ norm. As both the rounding and the projection are minimal distance operations (with respect to the $l_{1}$ norm), the distance between pairs of points on the two trajectories decreases monotonically until the method cycles, unable to further decrease the distance. If the distance is reduced to zero before cycling, then a feasible point has been obtained. Otherwise a random move is used to restart the method from a new point. In the original FP [17], the only bias of the method toward points of good objective value is the starting point, so the random restarts threaten to escape the good quality area. Thus, the feasibility pump uses a sophisticated restart scheme, attempting to displace the current point in an economic way by "minor perturbations," and performs a "major restart" only in the presence of further failures.

* Received by the editors February 7, 2011; accepted for publication (in revised form) May 15, 2012; published electronically July 19, 2012. This research was supported by ARC Discovery grant DP0987445.

http://www.siam.org/journals/siopt/22-3/82359.html

${ }^{\dagger}$ Faculty of Science and Information Technology, School of Mathematical and Physical Sciences, University of Newcastle, Callaghan NSW 2308, Australia (Natashia.Boland@newcastle.edu.au, Faramroze.Engineer@newcastle.edu.au).

${ }^{\ddagger}$ School of Mathematical and Geospatial Sciences, RMIT, Melbourne 3001, Victoria, Australia (andy.eb@rmit.edu.au, tsouk@mit.edu). 
The FP method demonstrates encouraging performance on a large class of test problems but behaves better for binary problems than for general integer variables. Thus, [5] first deal with the binary variables in isolation, treating general integers as continuous variables. General integer variables are treated as integer in a subsequent second round only after a point satisfying integrality for all binary variables is obtained. In [1] the objective feasibility pump (OFP) is proposed, where the projection problem is modified to include an objective function term, while in [18] simple rounding is substituted by a sophisticated constraint propagation approach. The latter makes use of information about the LP feasible region, with rounding done one variable at a time, while exploiting constraint information to disqualify unacceptable values. A modified penalty function is used in [26] that implicitly promotes a bias toward fixing more variables at integral values during the projection step.

The numerical results presented in [1] indicate that adding an objective term to the projection problem is beneficial to the quality of the solution, while not compromising computation time too much. Constraint propagation [18] is shown to further improve solution quality while also decreasing the failure rate of the method. The modified penalty function of [26] yields an improvement in efficiency and number of iterations relative to the original FP [17]. Recently the FP has been successfully extended to nonlinear problems; see, for example, $[8,10,11]$, which focus on the challenge introduced by nonlinear (and nonconvex) constraints.

In this paper, we explore the relationship between generalized forms of the FP and proximal point algorithms, in the context of linearly constrainted (possibly nonlinear) integer programs (see, for example, $[9,25]$ ). In doing so, we provide insights about the properties of the points generated by the FP method and of its mathematical behavior. Aside from the OFP, methods that improve the original FP can be characterized as either putting some of the LP into the rounding step (e.g., constraint propagation in [18]) or putting some integrality into the projection step [26]. Here we also propose to explore the latter, but in a different way: by incorporating integer cutting planes into the LP projection step.

We consider the general nonlinear MIP

$$
\begin{array}{ll}
\min & g(x) \\
\text { s.t. } & A x \geq b, \quad x_{\mathcal{I}} \in \mathbf{I},
\end{array}
$$

where $g: \mathbf{R}^{n} \rightarrow \mathbf{R}$ is a possibly nonlinear continuous function, $\mathcal{I}$ is the index set of integer variables, and $\mathbf{I}=\mathbf{Z}^{m}$, where $m=|\mathcal{I}|$. The remaining variables, denoted by $x_{\mathcal{R}}$, are allowed to vary over $\mathbf{R}^{n-m}$; the nonlinear MIP feasible set is $F:=\left\{x \in \mathbf{R}^{n} \mid A x \geq b, x_{\mathcal{I}} \in \mathbf{I}\right\}$, where $A$ and $b$ are assumed rational. We will refer to integral points as being those points $x \in \mathbf{R}^{n}$ for which $x_{\mathcal{I}} \in \mathbf{I}$. Although the objective may be nonlinear, throughout we will refer to the feasible set with integrality relaxed as the $L P$ feasible set, defined as $F_{L P}:=\left\{x \in \mathbf{R}^{n} \mid A x \geq b\right\}$.

In this paper we will show that the FP heuristic can be interpreted as a kind of proximal point method, by comparing the FP with the primal-proximal heuristic introduced in [12]. Our analysis focuses on the "inner loop" of the FP method, during which the two trajectories of rounded and projected points are calculated, before cycling necessitates a perturbation or restart. We call this the pumping process and the points generated by rounding during this process the discrete proximal point iterates. Each of these points is associated with the LP feasible point it was rounded from: we call these the LP feasible iterates. The key departure of the FP method from standard proximal point methods is the rounding step, which in effect is a projection 
onto the integer points, giving a point minimizing $I(x):=\min \left\{\left\|x_{\mathcal{I}}-y_{\mathcal{I}}\right\|_{1} \mid y_{\mathcal{I}} \in \mathbf{I}\right\}$. Thus the rounding step implicitly calculates the measure of lack of integrality given by $I(x)$, where $x$ is an LP feasible iterate. One of the important results of our analysis in this paper is that LP feasible iterates generated during the pumping process converge to a local minimum of the function $g+r I$ on $F_{L P}$, where $r>0$ is a parameter that weights the importance of the two conflicting objectives. Thus local minimization of the objective $g+r I$ over $F_{L P}$ is associated with cycling events in the FP method.

In [24] and [26] pure integer programs (IP) were studied and it was noted that the global minimizers of various penalized continuous problems over $F_{L P}$ coincide with the global optimal solutions of the original IP. In this paper we will show that a similar interpretation of the original FP [17] and the OFP [1] can be made for an MIP. Thus if the pumping process of an FP method can be started in the right region (and with the right value for the parameter $r$ ), it will converge to an optimal solution of the MIP. Interestingly, FP methods do contain a mechanism for restarting the pumping process.

When the FP method detects cycling it performs either a "major" or a "minor" restart, both using some form of randomization. These restarts form the outer loop of iterates of the FP. We provide numerical results which show that the repeated use of major restarts in particular correlates well with cases in which poorer solution quality is obtained by the FP method. Our analysis explains that cycling occurs because local optimality of the function $g+r I$ over $F_{L P}$ has been obtained and hence suggests some alternative ways to overcome this problem when this local minimum is not integral. Two approaches to escaping these undesirable local optimum present themselves: effect a change on either the objective function or the feasible region $F_{L P}$. The former can be achieved by varying the weighting parameter $r>0$; we plan to consider this approach in future work. In this paper, we focus on the latter and propose to alter the feasible region via the introduction of cuts to remove the local, nonintegral minimum at which the cycling has occurred. We give encouraging numerical experiments which demonstrate the potential for this cutting strategy. We show the strategy tends to delay the use of major restarts and leads to improvements in the quality of the feasible integral solutions found.

The paper is structured as follows. In section 2 we reinterpret the inner iterates of the FP as a kind of a proximal point iteration and discuss the connection this interpretation has to the primal-proximal heuristic introduced in [12]. As we require more general versions of some results of [12, sections 1.3 and 2] we provide extensions of these results for our nonlinear MIP in section 3. In particular, we show that quite general coercive penalties can be used in place of the $l_{2}$ norm which was used in [12] in order to exploit Lagrangian relaxation techniques. As the FP exploits LP relaxations our analysis departs from that of [12] significantly at this point and in section 4 we complete the development begun in section 2 by showing how the minimization of $g+r I$ over $F_{L P}$ results from such proximal point iterates (the "pumping process"). In section 5 cycling is shown to be associated with the failure to locate an integral LP feasible point at a local minimum of $g+r I$. A modification of the OFP algorithm of [18] is proposed in section 6. Here extensive numerical testing is undertaken in which we selectively introduce cuts to prevent cycling instead of the sole use of minor perturbations.

2. FP, proximal points, and concave penalties. In this section we draw out some comparisons and contrasts with recent approaches that convert integer programs to nonlinear continuous optimization problems. Consider the general nonlinear 
MIP (1.1). Proximal point approaches [12] consider regularizing this problem using a penalty $\rho(x)$ to obtain a continuous optimization problem,

$$
\begin{gathered}
\min _{y} \varphi_{r}(y), \quad \text { where } \\
\varphi_{r}(y):=\min \{g(x)+r \rho(x-y) \mid x \in F\} .
\end{gathered}
$$

Regularization is a common tool used in nonlinear and nonsmooth optimization. In a sense, MIPs are highly nonsmooth, as their domains are disconnected. Regularization in the form of (2.2) can be seen as a mathematical tool to construct, in a systematic way, an equivalent continuous problem, with smoother functions. The results can have attractive properties, as we will see in sections 3 and 4 .

The analysis of the problem (2.1) when $\rho$ is the Euclidean $l_{2}$ norm was carried out in some detail in [12]. The main property that arises is that integrality of local minima of (2.1) is ensured and do often correspond to the feasible solutions of (1.1). Indeed $y_{0}$ (where $\left.\left(y_{0}\right)_{\mathcal{I}} \in \mathbf{I}\right)$ is a local minimum of $\varphi_{r}$ exactly when $x=y_{0}$ is the solution to the minimization problem $\varphi_{r}\left(y_{0}\right)$.

Since the calculation of the objective $\varphi_{r}$ is as difficult as the original MIP, in [12] Lagrangian relaxation is used to obtain a solvable relaxed problem. This gives an underestimate $\tilde{\varphi}_{r}$ of $\varphi_{r}$. Unfortunately the local minima of the relaxation $\tilde{\varphi}_{r}$ are not assured to be integral. In section 3 of [12], approaches to varying the parameter $r>0$ to obtain feasible integral points are considered, but the results are inconclusive and the tone of [12] is quite negative in this respect. In the final section of [12] binary problems are addressed and a penalty used to enforce integrality, but no role for rounding is considered.

The OFP, by contrast, drops the integrality constraint in (2.2) and takes $\rho$ to be an $l_{1}$ norm, so that a relaxation of $\varphi_{r}$ when $g(x)=c^{T} x$ can be calculated as a linear program. Thus we arrive at the problem

$$
\begin{gathered}
\min _{y} \varphi_{r}^{L P}(y), \quad \text { where } \\
\varphi_{r}^{L P}(y):=\min \left\{g(x)+r\|x-y\|_{1} \mid x \in F_{L P}\right\} .
\end{gathered}
$$

Proximal point algorithms seek local optima by a kind of fixed point iteration, where the center $y$ is replaced at each step by the optimal solution of the regularization problem (e.g., of (2.4)). FP methods also take this approach, but to try to encourage integrality of a solution, a new center at $y_{0}$ with $\left(y_{0}\right)_{\mathcal{I}} \in \mathbf{I}$ is sought at each iterate. If $x=y_{0}$ is the solution to the minimization problem, the FP method is clearly finished, having obtained a feasible integral point. Otherwise, the nonintegral solution of (2.4) is rounded to the nearest integral point in order to provide the new center for the next iteration.

Since FP methods use the $l_{1}$ norm, the analysis of [12] does not directly apply. Thus in order to establish similar properties, we in section 3 extend these results and in doing so prove they hold for general class of coercive functions $\rho$, including the $l_{1}$ norm as a special case. This allows us to show that regularization preserves the global minimizers (which are the optimal solutions of the MIP) and retains the same local minimizers (which correspond to the feasible integrals solutions of the MIP). These correspondences are established in section 3 for our general nonlinear MIP.

In section 5 we show that the pumping process applied in an OFP setting finds local optima of $g+r I$ over $F_{L P}$ and so the function $I$ acts as a penalty for the deviation from integrality. The original FP is essentially the limiting version of the scheme for $r \rightarrow \infty$. Thus the pumping phase of the FP implicitly performs a minimization 
of a concave nonlinear integrality penalty via a combination of projective LPs and roundings. Our results provide some additional insights. For example, if the OFP finishes early without major restarts, it appears likely that the resulting solution will be both an optimal for the original MIP and a global minimum of $g+r I$.

This approach is distinct from that proposed in [24], where piecewise concave penalties are used to obtain an exact penalization for IPs and is motivated by the long observed connection that binary programs have to concave minimization. This is exploited in [26] to provide an interpretation of the FP for the case of binary problems. Motivated by the observation of [14] they note that the original FP [17] for binary problems can be interpreted as a Frank-Wolfe method [7, section 2.2.2] as applied to a nondifferentiable concave function over the feasible set $F_{L P}$. They develop a theory that relates the global minima of these continuous penalized problems to the solution of an IP. We are able to provide similar results in section 5 , thus showing a connection of our approach to that used in [24].

3. General properties of regularizations. In this section we generalize key results of [12]. The generalization takes two forms: we use a general class of coercive penalty $\rho$ instead of $\rho=\|\cdot\|_{2}$ and give results for mixed rather than pure IPs. This yields results for $\rho=\|\cdot\|_{1}$ that apply to the FP heuristic for MIPs. In particular, we show that the regularization (2.1) has global and local minimizers closely related to the optimal and feasible points of the original MIP. We posit the following assumptions.

Assumption. A continuous function $\rho: \mathbf{R}^{n} \rightarrow \mathbf{R}$ that satisfies the following conditions is called an integer compatible regularization function (ICRF) iff the following hold:

Condition 1: $\rho(x) \geq 0$ for all $x$ and $x=0$ iff $\rho(x)=0$.

Condition 2: If $\gamma \in(0,1)$ then $\rho(\gamma x)<\rho(x)$ for all $x \neq 0$.

Condition 3: There exists a continuous, strictly increasing function $s(\cdot): \mathbf{R}_{+} \rightarrow$ $\mathbf{R}_{+}$and a $\bar{K}$ such that for all $K<\bar{K}$ we have $\rho(x) \leq K$ implies $\|x\|_{1} \leq s^{-1}(K)$ for the $l_{1}$ norm $\|\cdot\|_{1}$ on $\mathbf{R}^{n}$.

Remark 3.1. These properties are deliberately chosen to be general enough to allow all the theory to cover the case of any norm on $\mathbf{R}^{n}$ and for most of the theory to encompass the cases of some concave penalties already introduced in the literature for binary problems. Recall that in finite dimensions all norms are equivalent to the $l_{1}$ norm, i.e., for all norms $\|\cdot\|$ there exists constants $A, B>0$ such that $A\|\cdot\|_{1} \geq$ $\|\cdot\| \geq B\|\cdot\|_{1}$. With this in mind it is clear how these assumptions relate to equivalent norms.

In [26] and [24] penalty functions were generated as follows:

$$
\rho(x):=\sum_{i} p\left(\left|x_{i}\right|\right),
$$

where $p: \mathbf{R}_{+} \rightarrow \mathbf{R}_{+}$is a concave, strictly increasing function. Some examples taken from [26] that were used explicitly for binary problems are

$$
\begin{aligned}
& p(t)=\log (t+\alpha)-\log \alpha, \\
& p(t)=-[t+\alpha]^{-q}+\alpha^{-q}, \\
& p(t)=1-\exp (-\alpha t), \\
& p(t)=[1+\exp (-\alpha t)]^{-1}-\frac{1}{2}
\end{aligned}
$$

for some $\alpha, q>0$. We wish to include these functions in our analysis in order to make some comparisons with the approaches in [26] and [24]. It is interesting to note 
that the case when $p(x)=x$ corresponds to $p$ being both simultaneously concave and convex and to $\rho(\cdot)=\|\cdot\|_{1}$.

The following justifies the use of the Conditions 1 to 3 above for penalties of the form (3.1).

Proposition 3.2. Suppose $\rho$ is defined via (3.1) with $p(0)=0, p(t)>0$ for $t \neq 0$ and $p$ concave, continuous, and strictly increasing function. Then $\rho$ is an integer compatible regularization function. In particular when the Range $p=[0,+\infty)$ we may take $\bar{K}=+\infty$.

Proof. Suppose

$$
\rho(x)=\sum_{i} p\left(\left|x_{i}\right|\right) \leq K
$$

then for each $i$ we have $\left|x_{i}\right| \leq p^{-1}(K)$ when $K \in$ Range $p$. As Range $p$ contains an interval of the type $[0, b)$ we may take $0<K<\bar{K}:=b$. In particular, when $b=+\infty$ we can take $\bar{K}=+\infty$. Consequently

$$
\|x\|_{1} \leq \sqrt{n}\|x\|_{\infty} \leq \sqrt{n} p^{-1}(K)
$$

and hence $\rho$ satisfies Condition 3 with $s(\cdot):=\frac{1}{\sqrt{n}} p(\cdot)$. Clearly $\rho \geq 0$ and $\rho(x)=0$ iff $\|x\|_{1}=0$. As $s$ is strictly increasing $\rho(\gamma x)=\sum_{i} p\left(\gamma\left|x_{i}\right|\right)<\sum_{i} p\left(\left|x_{i}\right|\right)=\rho(x)$.

Let

$$
f(x):=\left\{\begin{array}{cc}
g(x) & \text { if } A x \geq b, x_{\mathcal{I}} \in \mathbf{I} \\
+\infty & \text { otherwise }
\end{array}\right.
$$

where $g: \mathbf{R}^{m} \rightarrow \mathbf{R}$ is a continuous function. Then

$$
\begin{aligned}
\varphi_{r}(y) & =\inf \left\{g(x)+r \rho(x-y) \mid A x \geq b, x_{\mathcal{I}} \in \mathbf{I}\right\} \\
& =\inf \left\{f(x)+r \rho(x-y) \mid x \in \mathbf{R}^{n}\right\} .
\end{aligned}
$$

Define

$$
f_{r}\left(x, y_{0}\right):=f(x)+r \rho\left(x-y_{0}\right) \quad \text { for all } x \in \mathbf{R}^{n}
$$

and so $\varphi_{r}\left(y_{0}\right)=\inf \left\{f_{r}\left(x, y_{0}\right) \mid x \in \mathbf{R}^{n}\right\}$. Note that as $g$ is continuous and the feasible region $F:=\left\{x \mid A x \geq b, x_{\mathcal{I}} \in \mathbf{I}\right\}$ is a closed set it follows that $f: \mathbf{R}^{m} \rightarrow \overline{\mathbf{R}}$ is a lower semicontinuous function. We also observe that as $\varphi_{r}$ is an infimum of a family of continuous functions $y \mapsto f(x)+r \rho(x-y)$ it is, at worst, an upper semicontinuous function of the variable $y$. We can claim more under mild conditions. Denote the epigraph epi $f:=\{(x, \alpha) \mid f(x) \leq \alpha\}$ and note that epi $h \supseteq$ epi $f+$ epi $r \rho$ iff $h(y) \leq f(x)+r \rho(x-y)$ for all $x, y \in \mathbf{R}^{n}$. Thus geometrically epi $\varphi_{r}$ is the largest extended real valued function whose epigraph contains the sum epi $f+\operatorname{epi} r \rho$.

Theorem 3.3. Suppose $g: \mathbf{R}^{n} \rightarrow \mathbf{R}$ is continuous and the feasible region $F$ is closed and nonempty. Suppose in addition that the regularized function $\varphi_{\bar{r}}\left(y_{0}\right)>$ $-\infty$ for some $y_{0}$ and that $\rho$ is an ICRF that is also Lipschitz continuous (with a global Lipschitz constant). Then $\varphi_{r}$ is finitely valued for $r \geq \bar{r}$ and globally Lipschitz continuous.

Proof. Clearly $\varphi_{r}(y) \geq \varphi_{\bar{r}}(y)$ for all $y$ and $r \geq \bar{r}$. Thus when $\varphi_{\bar{r}}(y)>-\infty$ for all $y$, then $\varphi_{r}(y)>-\infty$ for all $r \geq \bar{r}$. Moreover as $\varphi_{r}(y) \leq g(x)+r \rho(x-y)<+\infty$ for any $x \in F$ finiteness of $\varphi_{r}$ follows. Thus if we can show $\varphi_{r}(y)$ is bounded away from negative infinity for all $y$, then we would have shown it to be finite valued. 
Let $C_{\mu}$ denote the cone epi $\mu\|\cdot\|$. Note next that the Lipschitz continuity property of $\rho$ corresponds to the existence of a Lipschitz constant $\mu>0$ such that epi $\rho+C_{\mu} \subseteq$ epi $\rho$. Now epi $\rho+C_{\mu} \subseteq$ epi $\rho$ in turn implies epi $r \rho+C_{r \mu} \subseteq \operatorname{epi} r \rho$ with $\operatorname{int} C_{r \mu} \neq \emptyset$ for all $r$.

Suppose $\varphi_{r}(y)=-\infty$ for some $y$; then

$$
(y,-n) \in \operatorname{epi} f+\operatorname{epi} r \rho
$$

for all $n \in \mathbf{Z}^{+}$and so (using $\operatorname{int} C_{r \mu} \neq \emptyset$ )

$$
\begin{aligned}
\mathbf{R}^{n+1} \subseteq \bigcup_{n=1}^{\infty}\left((y,-n)+C_{r \mu}\right) & \subseteq(\operatorname{epi} f+\operatorname{epi} r \rho)+C_{r \mu} \\
& \subseteq \operatorname{epi} f+\left(\operatorname{epi} r \rho+C_{r \mu}\right) \subseteq \operatorname{epi} f+\operatorname{epi} r \rho \subseteq \operatorname{epi} \varphi_{r},
\end{aligned}
$$

implying $\varphi_{r} \equiv-\infty$, contradicting $\varphi_{r}\left(y_{0}\right)$ finite. Let $\varphi_{r}(y)<\alpha$ and take $x$ such that $f(x)+r \rho(x-y)<\alpha$. Let $z \in \mathbf{R}^{n} ;$ then

$$
\begin{aligned}
\varphi_{r}(z) & \leq f(x)+r \rho(x-z) \leq(f(x)+r \rho(x-y))+r(\rho(x-z)-\rho(x-y)) \\
& <\alpha+r(\rho(x-z)-\rho(x-y)) \leq \alpha+r \mu\|z-y\|,
\end{aligned}
$$

where we have used the Lipschitz continuity of $\rho$ again. As this holds for all $\alpha>\varphi_{r}(y)$ we have $\varphi_{r}(z) \leq \varphi_{r}(y)+r \mu\|z-y\|$. As $y, z \in \mathbf{R}^{n}$ are arbitrary we are finished.

Remark 3.4. The conditions of Theorem 3.3 are satisfied for any norm and all the concave penalties formed via (3.1) using the various choices for the function $p$.

The following results are a generalization of the results of [12, section 1] and these form a basic tool in the proof of subsequent properties. The most important property is Property 3, which roughly says that local minima of $\varphi_{r}$ are not only integral but correspond to "fixed points" of the mapping $y_{0} \mapsto \arg \min \left\{f_{r}\left(x, y_{0}\right) \mid x \in \mathbf{R}^{n}\right\}$.

Lemma 3.5. Posit the assumption that $\rho$ is an ICRF.

1. For all $y \in \mathbf{R}^{n}$ the function $r \mapsto \varphi_{r}(y)$ is nondecreasing.

2. $\varphi_{r}(y) \leq f(y)$ for all $y \in \mathbf{R}^{n}$ for all $r \geq 0$.

3. Assume $f$ is bounded from below. Then there exists $\bar{r}>0$ such that if $y_{0}$ is a local minimum of $\varphi_{r}$ for some $\bar{r}<r$, then $x=y_{0}$ is the unique minimum point of $f_{r}\left(\cdot, y_{0}\right)$ in

$$
\varphi_{r}\left(y_{0}\right)=\inf \left\{f_{r}\left(x, y_{0}\right) \mid x \in \mathbf{R}^{n}\right\} .
$$

In particular $\varphi_{r}\left(y_{0}\right)=f\left(y_{0}\right)$ and $y_{0}$ is integral. Moreover, if $\bar{K}=+\infty$ in the definition of ICRF, then $\bar{r}=0$.

4. Local minima of $\varphi_{r}$ are also local minima of $\varphi_{r^{\prime}}$ for $r^{\prime} \geq r$.

Proof. We show part 3 only, as it is the critical observation; the other observations follow in a similar way to the results of [12, section 1]. As $f$ is lower semicontinuous and bounded from below and Condition 3 of the definition of a integer compatible regularization function holds, implying the existence of function $s$, we have

$$
\begin{aligned}
& \left\{x \mid g(x)+r \rho\left(x-y_{0}\right) \leq K, A x \geq a, x_{\mathcal{I}} \in \mathbf{I}\right\} \\
& \subseteq\left\{x \mid \min _{z} f(z)+r \rho\left(x-y_{0}\right) \leq K\right\} \subseteq\left\{x \mid\left\|x-y_{0}\right\|_{1} \leq s^{-1}\left(\frac{K-\min _{z} f(z)}{r}\right)\right\},
\end{aligned}
$$

Copyright $@$ by SIAM. Unauthorized reproduction of this article is prohibited. 
which is bounded and nonempty for any $K>\min _{z} f(z)$ and $r>0$ sufficiently large so that

$$
\left(\frac{K-\min _{z} f(z)}{r}\right)<\bar{K}
$$

Let $y_{0}$ be a local minimum of $\varphi_{r}$, so there exists an integral point $x_{0} \in F$ and a neighborhood of $y_{0}$ denoted by $B_{\varepsilon}\left(y_{0}\right)$ (for some $\varepsilon>0$ ) such that

$$
f\left(x_{0}\right)+r \rho\left(x_{0}-y_{0}\right)=\varphi_{r}\left(y_{0}\right) \leq \varphi_{r}(y) \leq f\left(x_{0}\right)+r \rho\left(x_{0}-y\right)
$$

for any $y \in B_{\varepsilon}\left(y_{0}\right)$. Hence $\rho\left(x_{0}-y_{0}\right) \leq \rho\left(x_{0}-y\right)$ for any $y \in B_{\varepsilon}\left(y_{0}\right)$. Assume that $\rho\left(x_{0}-y_{0}\right)>0$. Take $y=y_{0}+t\left(x_{0}-y_{0}\right)$ and so if $x_{0}-y_{0} \neq 0$ we have for $t$ sufficiently small

$$
\rho\left(x_{0}-y_{0}\right) \leq \rho\left(x_{0}-y\right)=\rho\left((1-t)\left(x_{0}-y_{0}\right)\right)<\rho\left(x_{0}-y_{0}\right),
$$

which is a contradiction when $0<t<1$ under Condition 3 if $\rho\left(x_{0}-y_{0}\right)>0$. Thus $\rho\left(x_{0}-y_{0}\right)=0$ and so $x_{0}=y_{0}$ and $\left(y_{0}\right)_{\mathcal{I}} \in \mathbf{I}$ with $\varphi_{r}\left(y_{0}\right)=f_{r}\left(y_{0}, y_{0}\right)=f\left(y_{0}\right)$.

The relationship between $\inf _{y \in \mathbf{R}^{n}} \varphi_{r}(y)$ and $\inf \left\{g(x) \mid A x \geq b, x_{\mathcal{I}} \in \mathbf{I}\right\}$ is developed in the next result, which is essentially Theorem 1.5 of [12] and provided for completeness. This result demonstrates that there is a direct correspondence between the local and global minimizers of the nonlinear MIP and the unconstrained continuous regularized problem $\varphi_{r}$.

TheOREM 3.6. Posit the assumption that $\rho$ is an ICRF. Then the minimization of $f$ and $\varphi_{r}$ are related as follows:

1. $\inf \left\{g(x) \mid A x \geq b, x_{\mathcal{I}} \in \mathbf{I}\right\}=\inf \left\{\varphi_{r}(y) \mid y \in \mathbf{R}^{n}\right\}$.

2. If $x^{*} \in \arg \min \left\{f(y) \mid y \in \mathbf{R}^{n}\right\}=\arg \min \left\{g(x) \mid A x \geq b, x_{\mathcal{I}} \in \mathbf{I}\right\}$, then $x^{*} \in \arg \min \left\{\varphi_{r}(y) \mid y \in \mathbf{R}^{n}\right\}$.

3. Suppose $\bar{r}$ is as specified in Lemma 3.5, part 3, and $f$ is bounded below. Then for $r>\bar{r}$ we have $y^{*}$ minimizes (resp., locally minimizes) $\varphi_{r}$, then $y^{*}$ minimizes (resp., locally minimizes) $f$.

Proof. Consider the first part. For any $x$ and $y$ in $\mathbf{R}^{n}$ we have $f_{r}(x, y) \geq f(x)$ and hence $\varphi_{r}(y) \geq \inf f$ and so $\inf \varphi_{r} \geq \inf f$. On the other hand, Lemma 3.5, part 2, gives $\inf \varphi_{r} \leq \inf f$ and equality. Suppose $x^{*} \in \arg \min \left\{f(x) \mid x \in \mathbf{R}^{n}\right\}$. Then using Lemma 3.5, part 2, again we have inf $\varphi_{r}=\inf f=f\left(x^{*}\right) \geq \varphi_{r}\left(x^{*}\right)$ and so $x^{*} \in \arg \min \left\{\varphi_{r}(y) \mid y \in \mathbf{R}^{n}\right\}$. Finally, posit the assumption of Lemma 3.5, part 3 , and suppose $y^{*}$ minimizes (resp., locally minimizes) $\varphi_{r}$; then $f\left(y^{*}\right)=\varphi_{r}\left(y^{*}\right) \leq$ $\varphi_{r}(y) \leq f(y)$ for all $y$ ( $y$ close to $\left.y_{0}\right)$.

The converse of Lemma 3.5, part 3, may be developed and this result establishes when the local minimizers of the regularized problem are actually strict local minimizers. As the results of this paper are in the main based only on the implication given in Lemma 3.5, part 3, we relegate the proof of the next result to an appendix.

Proposition 3.7. Suppose $g$ is bounded below on the feasible region $F, \rho$ is an ICRF, and $r>\bar{r}$ (as specified in Lemma 3.5, part 3). A point $y_{0}$ is a local minimum of $\varphi_{r}$ iff $x=y_{0}$ with $x_{\mathcal{I}} \in \mathbf{I}$ is a unique optimal solution of

$$
\varphi_{r}\left(y_{0}\right)=\min \left\{g(x)+r \rho\left(x-y_{0}\right) \mid A x \geq b, x_{\mathcal{I}} \in \mathbf{I}\right\} .
$$

Indeed if $y_{0}$ is a local minima of $\varphi_{r}$, then it is a strict local minimum of $\varphi_{r}$ in the following cases:

1. We have an IP (i.e., $m=n)$.

Copyright (c) by SIAM. Unauthorized reproduction of this article is prohibited. 
2. $\left(y_{0}\right)_{\mathcal{R}}$ is a strict local minimum of the continuous partial regularization

$$
G_{r}\left(y_{\mathcal{R}}\right):=\min _{z_{\mathcal{R}} \in \mathbf{R}^{n-m}}\left\{G_{y_{0}}\left(z_{\mathcal{R}}\right)+r \rho\left(z_{\mathcal{R}}-y_{\mathcal{R}}\right)\right\}
$$

of the function $G_{y_{0}}\left(z_{\mathcal{R}}\right):=f\left(\left(y_{0}\right)_{\mathcal{I}}, z_{\mathcal{R}}\right): \mathbf{R}^{n-m} \rightarrow \mathbf{R}$.

In general we can only assert that when $y_{0}$ is a local minimum of $\varphi_{r}$, then $\left(y_{0}\right)_{\mathcal{R}}$ must be a local minimum of $G_{r}$, while $\left(y_{0}\right)_{\mathcal{I}}$ is always a strict local minimum of $x_{\mathcal{I}} \mapsto \varphi_{r}\left(x_{\mathcal{I}},\left(y_{0}\right)_{\mathcal{R}}\right)$.

The next result completes our goal of relating the local minimizers of $\varphi_{r}$ to the feasible solution of the MIP defined in (1.1) and the global minimizers of the regularized problem $\varphi_{r}$ to the global minimizers of the original nonlinear MIP defined in (1.1). This is a generalization of Theorem 2.2 of [12]. Note that when $\rho$ is a norm or an appropriate cohersive function with Range $\rho=[0,+\infty$ ) (see Proposition 3.2), then one may take $\bar{K}=+\infty$ in the definition of ICRF.

THEOREM 3.8. Suppose $\rho$ is an ICRF. Then there exists an $\bar{r}>0$ such that for $r>\bar{r}$ any local minimum of $\varphi_{r}$ lies in the feasible region $F$. Moreover, the following hold:

1. For $r>\bar{r}$ large enough, the local minima of $\varphi_{r}$ are exactly the points of $F$.

2. Suppose that $\bar{K}=+\infty$ in the definition of ICRF (and so by Lemma 3.5 part 3 we may take $\bar{r}=0$ ). Then for $r>0$ small enough, the local minima of $\varphi_{r}$ are exactly the optimal solutions of

$$
\min \left\{g(x) \mid A x \geq b, x_{\mathcal{I}} \in \mathbf{I}\right\}
$$

in the following cases:

1. we have $m=n$, i.e., a pure IP, or

2. both $g$ and $\rho$ are convex functions.

Proof. Consider the first part. In view of Lemma 3.5, part 3, we only need show that if $y_{0} \in F$, then $y_{0}$ is a local minimum of $\varphi_{r}$ for $r$ sufficiently large. Consider $K:=\varphi_{r}\left(y_{0}\right)+2, r>\bar{r}$, sufficiently large so that via (3.7) we have the set

$$
F_{K}\left(y_{0}\right):=\left\{x \in \mathbf{R}^{m} \mid\left\{f(x)+r \rho\left(x-y_{0}\right)\right\} \leq K\right\}
$$

bounded. As $f$ is lower semicontinuous and $r g\left(\cdot-y_{0}\right)$ is continuous, their sum is also lower semicontinuous, implying $F_{K}\left(y_{0}\right)$ is a closed set. Next note that as $\varphi_{r}(y)$ is defined as an infimum of continuous functions $y \mapsto f(x)+r \rho(x-y)$ it is upper semicontinuous. Thus we may take $y$ sufficiently close to $y_{0}$ so that $\varphi_{r}(y)+1<$ $\varphi_{r}\left(y_{0}\right)+2:=K$ and for all $x \in F_{K}\left(y_{0}\right)$ with $x_{\mathcal{I}} \neq\left(y_{0}\right)_{\mathcal{I}}$, then

$$
\begin{aligned}
\rho(x-y)= & \rho\left(x-y_{0}\right)+\left[\rho(x-y)-\rho\left(x-y_{0}\right)\right] \\
\geq & \inf \left\{\rho\left(x-y_{0}\right) \mid x \in F_{K}\left(y_{0}\right) \text { with } x_{\mathcal{I}} \neq\left(y_{0}\right)_{\mathcal{I}}\right\} \\
& -\left[\rho\left(x-y_{0}\right)-\rho(x-y)\right] \\
= & \epsilon-\left[\rho\left(x-y_{0}\right)-\rho(x-y)\right]>0,
\end{aligned}
$$

where $\inf \left\{\rho\left(x-y_{0}\right) \mid x \in F_{K}\left(y_{0}\right)\right.$ with $\left.x_{\mathcal{I}} \neq\left(y_{0}\right)_{\mathcal{I}}\right\}:=\epsilon>0$. Take $r>0$ sufficiently large so that $\frac{\epsilon}{2}>\frac{\Gamma}{r}>0$, where

$\Gamma:=\max \left\{f(x)-f\left(x^{\prime}\right) \mid x, x^{\prime} \in F_{K}\left(y_{0}\right)\right\}=\max \left\{g(x)-g\left(x^{\prime}\right) \mid x, x^{\prime} \in F \cap F_{K}\left(y_{0}\right)\right\}$,

which is finite as $g$ is continuous, $F_{K}\left(y_{0}\right)$ is compact, and $F$ is closed. We will assume we take $y$ sufficiently close to $y_{0}$ so that at least $\left[\rho\left(x-y_{0}\right)-\rho(x-y)\right]<\frac{\epsilon}{2}$ and hence $\rho(x-y) \geq \frac{\epsilon}{2}>\frac{\Gamma}{r}$. 
Then for $x \in F_{K}\left(y_{0}\right)$ with $x_{\mathcal{I}} \neq\left(y_{0}\right)_{\mathcal{I}}$ we have (as $y_{0} \in F_{K}\left(y_{0}\right)$ )

$$
\begin{aligned}
f(x)+r \rho(y-x) & =\left\{f(x)-f\left(y_{0}\right)+r \rho(x-y)\right\}+f\left(y_{0}\right) \\
& \geq-\Gamma+r \frac{\epsilon}{2}+f\left(y_{0}\right) \geq-\Gamma+r \frac{\Gamma}{r}+f\left(y_{0}\right)=f\left(y_{0}\right) \geq \varphi_{r}\left(y_{0}\right) .
\end{aligned}
$$

Next note that if $x \in F_{K-1}(y)$ and we have $y$ is sufficiently close to $y_{0}$ so that $r\left|\rho(x-y)-\rho\left(x-y_{0}\right)\right| \leq 1$, then

$$
\begin{aligned}
K-1 & \geq f(x)+r \rho(x-y) \\
& =f(x)+r \rho\left(x-y_{0}\right)+r\left(\rho(x-y)-\rho\left(x-y_{0}\right)\right) \\
& \geq f(x)+r \rho\left(x-y_{0}\right)-1
\end{aligned}
$$

and so $F_{K-1}(y) \subseteq F_{K}\left(y_{0}\right)$. Consequently

$$
\begin{aligned}
\varphi_{r}(y) & =\inf \left\{f(x)+r \rho(y-x) \mid x \in F_{K-1}(y)\right\} \\
& \geq \varphi_{r}\left(y_{0}\right) .
\end{aligned}
$$

For the second part we argue again along the lines of Theorem 2.2 of [12]. Let $y_{0}$ be a local minimizer of $\varphi_{r}$ and we already know that $y_{0} \in F$ and that $\varphi_{r}\left(y_{0}\right)=f\left(y_{0}\right)$ and need to show that $y_{0}$ is optimal for the MIP for $r$ small. Denote $V^{*}$ the optimal solutions of the MIP. Place $V_{\mathcal{I}}^{*}:=\left\{x_{\mathcal{I}} \mid x \in V^{*}\right\}$,

$$
\begin{aligned}
v^{+} & =\min \left\{f(x) \mid x \in F \cap\left\{x \mid x_{\mathcal{I}} \notin V_{\mathcal{I}}^{*}\right\}\right\} \quad \text { and } \\
v^{*} & =\min \{f(x) \mid x \in F\} .
\end{aligned}
$$

Now as the variables are partly integral valued we have $v^{+}>v^{*}$ as $x_{\mathcal{I}} \neq y_{\mathcal{I}}$ if $y \in V^{*}$ and $x_{\mathcal{I}} \in V_{\mathcal{I}}^{*}$. Let $K=v^{*}+1$ and suppose contrary to assertion that $y_{0} \notin V^{*}$ for any $r>0$. Denote

$$
D:=\max \left\{\rho\left(x-y_{0}\right) \mid x_{\mathcal{I}} \neq\left(y_{0}\right)_{\mathcal{I}}, x \in V^{*}\right\} .
$$

When $D=0$ we immediately have $\left(y_{0}\right)_{\mathcal{I}} \in V_{\mathcal{I}}^{*}$. Otherwise $D>0$; then we choose $0<r<\left(v^{+}-v^{*}\right) / D$ and we have for any $x^{*} \in V^{*}$

$$
\begin{aligned}
f\left(y_{0}\right) & =\varphi_{r}\left(y_{0}\right) \leq f\left(x^{*}\right)+r \rho\left(x^{*}-y_{0}\right) \\
& \leq f\left(x^{*}\right)+r D<f\left(x^{*}\right)+v^{+}-v^{*}=v^{+} .
\end{aligned}
$$

As $f\left(y_{0}\right)<v^{+}$we must have $\left(y_{0}\right)_{\mathcal{I}} \in V_{\mathcal{I}}^{*}$ once again. If $n=m$ (i.e., we have an IP) we are finished as then $y_{0} \in V^{*}$.

In the final case we assume contrary to the theorem that $\left(y_{0}\right)_{\mathcal{R}} \notin V_{\mathcal{R}}^{*}$ and $v^{*}<$ $f\left(\left(y_{0}\right)_{\mathcal{I}},\left(y_{0}\right)_{\mathcal{R}}\right)=f\left(y_{0}\right)$. As $\left(y_{0}\right)_{\mathcal{I}} \in V_{\mathcal{I}}^{*}, y_{0} \in F$, and $y_{0} \notin V^{*}$, we must have the existence $\bar{y}_{\mathcal{R}}$ such that $\left(\left(y_{0}\right)_{\mathcal{I}}, \bar{y}_{\mathcal{R}}\right) \in F$ and $v^{*}=f\left(\left(y_{0}\right)_{\mathcal{I}}, \bar{y}_{\mathcal{R}}\right)<f\left(\left(y_{0}\right)_{\mathcal{I}},\left(y_{0}\right)_{\mathcal{R}}\right)$. Thus by Lemma 3.5, part 3, we have

$$
\begin{aligned}
\varphi_{r}\left(\left(y_{0}\right)_{\mathcal{I}}, \bar{y}_{\mathcal{R}}\right) & \leq f\left(\left(y_{0}\right)_{\mathcal{I}}, \bar{y}_{\mathcal{R}}\right) \\
& <f\left(\left(y_{0}\right)_{\mathcal{I}},\left(y_{0}\right)_{\mathcal{R}}\right)=\min _{y}\left\{f(y)+r\left(y-y_{0}\right)\right\}=\varphi_{r}\left(y_{0}\right) .
\end{aligned}
$$

For $t$ sufficiently small we have $(1-t) y_{0}+t\left(\left(y_{0}\right)_{\mathcal{I}}, \bar{y}_{\mathcal{R}}\right)$ in the neighborhood in which $y_{0}$ is the local minimum of $\varphi_{r}$. When $\rho$ and $g$ are convex we have $\varphi_{r}$ convex and so

$$
\begin{aligned}
\varphi_{r}\left((1-t) y_{0}+t\left(\left(y_{0}\right)_{\mathcal{I}}, \bar{y}_{\mathcal{R}}\right)\right) & \leq(1-t) \varphi_{r}\left(y_{0}\right)+t \varphi_{r}\left(\left(y_{0}\right)_{\mathcal{I}}, \bar{y}_{\mathcal{R}}\right) \\
& <(1-t) \varphi_{r}\left(y_{0}\right)+t \varphi_{r}\left(y_{0}\right)=\varphi_{r}\left(y_{0}\right),
\end{aligned}
$$

implying $y_{0}$ is not a local minimum of $\varphi_{r}$, a contradiction. 
4. The proximal point heuristic and relaxation of integrality. The relationship of the analysis above to the FP methods lies in the observation that an OFP iteration solves (2.4), i.e., solves the LP relaxation of problem (3.6) with $g(x)=c^{T} x$, and the integer compatible regularization function given by $\rho(x)=\|x\|_{1}$. At this juncture it is important to note that Range $\rho=[0,+\infty)$ so we may take $\bar{r}=0$, which will be the case for the remainder of the paper unless otherwise indicated. Recall the definition of $\varphi_{r}^{L P}$ from (2.4) and observe that

$$
\varphi_{r}^{L P}(y) \leq \varphi_{r}(y)
$$

for all $y$. When $g(x)=c^{T} x$ the problem of calculating $\varphi_{r}^{L P}(y)$ may be reformulated as an LP. Indeed this is the basis of the FP strategy. Calculating $\varphi_{r}^{L P}(y)$ is not so easy when $g$ is nonlinear, but we carry out the following analysis without any linearity assumptions on $g$, and this allows for the possibility of developing some other novel numerical method in the future.

Remark 4.1. The analysis of this section (and the next) can be carried out using a much more general form of an integer compatible regularization function $\rho$ than the $l_{1}$ norm. In particular, a general norm, or any other convex, integer compatible regularization function, would suffice. For example, if $g$ were quadratic, one could use $\rho(x)=\|x\|^{2}$ and then $\varphi_{r}^{L P}(y)$ could be solved as a quadratic program. As we wish to emphasis our comparison with the feasibility pump, these generalizations are not pursued here.

Placing

$$
h(x):=\left\{\begin{array}{cc}
g(x) & \text { if } A x \geq b, x \in \mathbf{R}^{n}, \\
+\infty & \text { otherwise }
\end{array}\right.
$$

we may write $\varphi_{r}^{L P}$ as a proximal point problem,

$$
\varphi_{r}^{L P}\left(y_{0}\right):=\inf _{x}\left\{h(x)+r\left\|x-y_{0}\right\|_{1}\right\},
$$

which is well known to define a convex, Lipschitz function of $y_{0}$ when $g$ is convex (see [12, Theorem 1.6] and references). The same proof technique as used in Lemma 3.5 shows that if $y_{0}$ is a local minimum of $\varphi_{r}^{L P}$, then $x=y_{0}$ is the unique minimum point of $h(\cdot)+r \rho\left\|\cdot-y_{0}\right\|_{1}$. Now in the event that $\left(y_{0}\right)_{\mathcal{I}} \in \mathbf{I}$, then clearly $\varphi_{r}^{L P}\left(y_{0}\right)=\varphi_{r}\left(y_{0}\right)$ and $x=y_{0}$ is the unique minimum point of $h(\cdot)+r \rho\left\|\cdot-y_{0}\right\|_{1}$ over $x$ constrained to $x_{\mathcal{I}} \in \mathbf{I}$. Furthermore, using (4.1) and the fact that $y_{0}$ is a local minimum of $\varphi_{r}^{L P}$ we have

$$
\varphi_{r}\left(y_{0}\right)=\varphi_{r}^{L P}\left(y_{0}\right) \leq \varphi_{r}^{L P}(y) \leq \varphi_{r}(y) \quad \text { for all } y \text { close to } y_{0} .
$$

Thus $y_{0}$ must also be a local minimum of $\varphi_{r}$. By Theorem 3.8 we have $y_{0} \in$ $\left\{A x \geq b, x_{\mathcal{I}} \in \mathbf{I}\right\}$ and moreover if $g$ is convex and $r$ is sufficiently small $y_{0}$ is actually optimal for (3.9). The OFP [1] increases $r$ at each step of the pumping process and so between major restarts the magnitude of $r$ is directly related to the iteration number in this pumping process. When the OFP terminates early, without any restarts, $r$ will be smaller than it would be if multiple restarts occurred. (Long durations of the pumping process are not observed in numerical tests.) As smaller values of $r$ are needed for the FP to generate optimal solutions (an observation that follows from Theorem 3.8, part 2), we hope that the FP's chances of generating an optimal point will be higher when we have fewer restarts. 
Proposition 3.7 indicates that we can detect in $\left(y_{0}\right)_{\mathcal{I}} \in \mathbf{I}$ a local minimizer of $\varphi_{r}$ by checking if $x_{\mathcal{I}}=\left(y_{0}\right)_{\mathcal{I}}(\in \mathbf{I})$ is a unique optimal solution of (3.8) or in our case it suffices to check if $x_{\mathcal{I}}=\left(y_{0}\right)_{\mathcal{I}}(\in \mathbf{I})$ is a unique optimal solution of (4.2) (for any fixed $r>0=\bar{r})$. Now when $y_{0} \in F:=\left\{A x \geq b, x_{\mathcal{I}} \in \mathbf{I}\right\}$ we have from Theorem 3.8 that $y_{0}$ is a local minimum of $\varphi_{r}$ for $r$ sufficiently large and hence $x=y_{0}$ is the unique optimal solution of (3.8). Is the same true for our relaxation (4.2)? The following partly answers this question.

TheOrem 4.2. Assume $g$ is continuous and bounded below on $\{A x \geq b, x \in \mathbf{I}\}$.

1. Suppose $r$ is sufficiently small and $y_{0}$ with $\left(y_{0}\right)_{\mathcal{I}} \in \mathbf{I}$ is a local minima of $\varphi_{r}^{L P}$. Then $y_{0}$ is an optimal solution of (3.9). In particular $x=y_{0}$ with $\left(y_{0}\right)_{\mathcal{I}} \in \mathbf{I}$ is the minimum of the problem

$$
\inf \left\{g(x)+r\left\|x-y_{0}\right\|_{1} \mid A x \geq b, x \in \mathbf{R}^{n}\right\} .
$$

2. Suppose $r$ is sufficiently large and $y_{0}$ with $\left(y_{0}\right)_{\mathcal{I}} \in \mathbf{I}$ is a local minima of $\varphi_{r}^{L P}$. Then $y_{0} \in F:=\left\{A x \geq b, x_{\mathcal{I}} \in \mathbf{I}\right\}$ and $x=y_{0}$ solves the problem (4.4). Conversely, when $g$ is convex and $y_{0} \in F$, then $x=y_{0}$ with $x_{\mathcal{I}} \in \mathbf{I}$ solves the problem (4.4) for $r$ sufficiently large.

Proof. Suppose $y_{0}$ with $\left(y_{0}\right)_{\mathcal{I}} \in \mathbf{I}$ is a local minima of $\varphi_{r}^{L P}$ then by (4.3) we have $y_{0}$ a local minimum of $\varphi_{r}$ and we may now apply Theorem 3.8 to obtain all but the last assertion.

Let $y_{0} \in F$ and as $h$ is convex, lower-semicontinuous, and bounded below with $\operatorname{dom} h=\left\{A x \geq b, x \in \mathbf{R}^{n}\right\}$ we may take $z_{0} \in \partial h\left(y_{0}\right) \neq \emptyset$. Then for all $x \in \operatorname{dom} h$ we have

$$
\begin{array}{cl}
h(x)-h\left(y_{0}\right) & \geq\left\langle z_{0}, x-y_{0}\right\rangle, \\
\text { implying } & g(x)+r\left\|x-y_{0}\right\|_{1} \geq g\left(y_{0}\right)+r\left\|x-y_{0}\right\|_{1}-\left\|z_{0}\right\|_{\infty}\left\|x-y_{0}\right\|_{1} \\
\text { or } & g(x)+r\left\|x-y_{0}\right\|_{1} \geq g\left(y_{0}\right)+\left(r-\left\|z_{0}\right\|_{\infty}\right)\left\|x-y_{0}\right\|_{1}>g\left(y_{0}\right)
\end{array}
$$

for all $x \in\left\{A x \geq b, x \in \mathbf{R}^{n}\right\}$ and all $x \neq y_{0}$ when $r>\left\|z_{0}\right\|_{\infty}$. Thus $x=y_{0}$ with $\left(y_{0}\right)_{\mathcal{I}} \in \mathbf{I}$ is the local minimum in (4.4).

One can of course directly test if a point $y_{0}$ is feasible, but if this test fails how can we improve our next test point? This is where the above comes into play. Suppose that we start with an initial feasible point $x_{0} \in F_{L P}$. Denote by $y_{0} \in$ $\arg \min \left\{\left\|x_{0}-y\right\|_{1} \mid y_{\mathcal{I}} \in \mathbf{I}\right\}$ the closest integral point. We solve (4.4) to obtain $x_{1} \in$ $\arg \min \left\{g(x)+r\left\|x-y_{0}\right\|_{1} \mid A x \geq b, x \in \mathbf{R}^{n}\right\}$ and find that if $x_{1} \neq y_{0}$, then $y_{0}$ fails to be a local minimum of $\varphi_{r}^{L P}$. Then when $x_{0} \neq x_{1}$ we have

$$
\begin{aligned}
g\left(x_{1}\right)+r\left\|x_{1}-y_{0}\right\|_{1} & \leq g\left(x_{0}\right)+r\left\|x_{0}-y_{0}\right\|_{1} \\
\text { or } \quad g\left(x_{1}\right) & \leq g\left(x_{0}\right)-r\left[\left\|x_{1}-y_{0}\right\|_{1}-\left\|x_{0}-y_{0}\right\|_{1}\right]<g\left(x_{0}\right),
\end{aligned}
$$

a decrease in objective value as long as

$$
\begin{aligned}
\left\|x_{1}-y_{0}\right\|_{1} & >\left\|x_{0}-y_{0}\right\|_{1}=I\left(x_{0}\right) \\
& :=\min \left\{\left\|x_{0}-y\right\|_{1} \mid y_{\mathcal{I}} \in \mathbf{I}\right\}=\left\|\left(x_{0}\right)_{\mathcal{I}}-\left(y_{0}\right)_{\mathcal{I}}\right\|_{1} .
\end{aligned}
$$

Inequality (4.6) indicates that this process does not cycle when (4.7) holds. Using (4.5), the fact that $I\left(x_{1}\right) \leq\left\|x_{1}-y_{0}\right\|_{1}$, and $\left\|x_{0}-y_{0}\right\|_{1}=I\left(x_{0}\right)$ we have for any two successive iterates

$$
g\left(x_{1}\right)+r I\left(x_{1}\right) \leq g\left(x_{0}\right)+r I\left(x_{0}\right) .
$$

Copyright $@$ ㅇ by SIAM. Unauthorized reproduction of this article is prohibited. 
Thus the proximal point iteration combined with rounding always attempts to decrease the weighted sum $g+r I$. It progressively decreases the weighted sum of objective and integrality gap measure $I$ at the feasible (possibly nonintegral points). As the point $x_{1}$ may not be integral and our test requires the new center at an integral point, we must round $x_{1}$ to get $\left(y_{1}\right)_{\mathcal{I}} \in \mathbf{I}$.

This observation somewhat justifies the strategy used in [1], where successive guesses of a suitable $\left(y_{0}\right)_{\mathcal{I}} \in \mathbf{I}$ are taken by solving the problem (4.4) and rounding the answer $x_{1}$ to a new estimate of $y_{1}$. This provides a new optimization problem $\varphi_{r}^{L P}\left(y_{1}\right)$ to solve for another $x_{2} \in\left\{A x \geq b, x \in \mathbf{R}^{n}\right\}=F_{L P}$, etc. The parameter $r$ starts off small and is increased as the algorithm proceeds, the object being to generate a "good" feasible solution to (3.9). Clearly one immediately terminates after a feasible $y_{k} \in\left\{A x \geq b, x_{\mathcal{I}} \in \mathbf{I}\right\}=F$ is obtained.

Our model algorithm for a nonlinear MIP FP method is thus

$$
\begin{aligned}
& x^{k+1} \in \arg \min \left\{g(x)+r^{k+1}\left\|x-y^{k}\right\|_{1} \mid A x \geq b, x \in \mathbf{R}^{n}\right\}, \\
& y^{k+1} \in \mathcal{R}\left(x^{k+1}\right):=\arg \min _{z: z_{\mathcal{I}} \in \mathbf{I}}\left\|x^{k+1}-z\right\|_{1} \text { (a rounding), }
\end{aligned}
$$

which we call the discrete proximal point algorithm. This gives a sequence of iterations that must have

$$
g\left(x^{k+1}\right)+r^{k+1} I\left(x^{k+1}\right) \leq g\left(x^{k}\right)+r^{k+1} I\left(x^{k}\right) .
$$

Forming a telescoping series for $k=0, \ldots, N$ we find

$$
g\left(x^{N}\right)-g\left(x^{0}\right) \leq-\sum_{k=0}^{N} r^{k+1}\left[I\left(x^{k+1}\right)-I\left(x^{k}\right)\right]
$$

and as $g$ is bounded below on $F_{L P}$ and $\left\{r^{k}\right\}$ is bounded away from zero we see that an infinitely long sequence of iterates would lead to $I\left(x^{k+1}\right)-I\left(x^{k}\right) \rightarrow 0$. In practice the failure to return a significant decrease in $I$ in subsequent iterates is associated with the cycling phenomena which we study next.

5. An interpretation of cycling in the FP. So far we have shown that the discrete proximal point algorithm is a descent algorithm for the function $g+r I$. In this section we want to explain why we can associate the nonintegral local minimizers of this function with the points around which the discrete proximal point algorithm will cycle. From this we may deduce that a local minimum over $F_{L P}$ is obtained from this iterative process.

For the purposes of this section we say that the discrete proximal algorithm cycles if $x^{k} \in \arg \min \left\{g(x)+r\left\|x-y^{k}\right\|_{1} \mid x \in F_{L P}\right\}$, where $y^{k} \in \mathcal{R}\left(x^{k}\right)$. This still leaves the choice of rounded point in $\mathcal{R}\left(x^{k}\right)$ ambiguous when the set contains more than one point, a case we will discuss further later. However, this definition does remove ambiguity about the possibility that $\min _{F_{L P}}\left\{g(\cdot)+r\left\|\cdot-y^{k}\right\|_{1}\right\}$ has multiple optimal solutions. In this case the cycling condition is easy to check by simply substituting in $x^{k}$. Alternative ways to describe cycling would be that $y^{k+1}=y^{k}$ or that the process fails to further reduce $g(\cdot)+r\left\|\cdot-y^{k}\right\|_{1}$ for an infinite number of iterations. The former is the cycling definition used in all implementations of the feasibility pump, while the latter would be the more accurate theoretical description of when you have lost all hope to make further progress without restarting. Our definition and that used in the feasibility pump would be equivalent if the mapping operations 
from $x^{k}$ to $y^{k}$ and vice versa were uniquely defined. Certainly if $y^{k+1}=y^{k}$, then $x^{k+1} \in \min _{F_{L P}}\left\{g(\cdot)+r\left\|\cdot-y^{k+1}\right\|_{1}\right\}$, so our definition of cycling applies. The converse is not necessarily true if $x^{k}$ has a nonunique rounding, in which case it depends purely on machine accuracy whether a component is rounded up or down; FP algorithms don't specify which way values extremely close to 0.5 should be rounded. Thus current FP algorithms may cycle through alternative roundings of the same LP feasible point, or alternative LP optima for the projection problem. In what follows, we discuss these issues and how they relate to local minima of $g+r I$.

Remark 5.1. The rounding $\mathcal{R}(x)$ is unique iff no components of $x$ have fractional parts 0.5. Also $|\mathcal{R}(x)|=1$, i.e., $y \in \mathcal{R}(x)$ is unique iff $y \in \mathcal{R}\left(x^{\prime}\right)$ for all $x^{\prime}$ sufficiently close to $x$.

We claim that the local minimizers of $g+r I$ over $F_{L P}$ correspond to points at which the discrete proximal point method cycles. First, we see that if a point at which the method cycles has a unique rounding, then it must be a local minimizer of $g+r I$. It is helpful for what follows to note that $y \in \mathcal{R}(x)$ iff $I(x)=\|x-y\|_{1}$.

Proposition 5.2. If the discrete proximal point algorithm cycles at $x^{k}$ and the rounding $y^{k} \in \mathcal{R}\left(x^{k}\right)$ is unique, then $x^{k}$ is a local minimizer of $g+r I$ over $F_{L P}$.

Proof. From Remark 5.1 and since the rounding of $x^{k}$ is unique, there is a neighborhood $N\left(x^{k}\right)$ such that for all points $x \in N\left(x^{k}\right), y^{k} \in \mathcal{R}(x)$. Suppose $x^{k}$ is not a local minimum of $g+r I$. Then there exists a point $x^{\prime}$ close to $x^{k}$, and certainly within $N\left(x^{k}\right)$, so that $g\left(x^{\prime}\right)+r I\left(x^{\prime}\right)<g\left(x^{k}\right)+r I\left(x^{k}\right)$. Now since $y^{k} \in \mathcal{R}\left(x^{k}\right)$,

$$
g\left(x^{k}\right)+r I\left(x^{k}\right)=g\left(x^{k}\right)+r\left\|x^{k}-y^{k}\right\|_{1} \leq g\left(x^{\prime}\right)+r\left\|x^{\prime}-y^{k}\right\|_{1}
$$

since the algorithm cycles at $x^{k}$, so $x^{k} \in \arg \min _{F_{L P}}\left\{g(\cdot)+r\left\|\cdot-y^{k}\right\|_{1}\right\}$. But $g\left(x^{\prime}\right)+$ $r\left\|x^{\prime}-y^{k}\right\|_{1}=g\left(x^{\prime}\right)+r I\left(x^{\prime}\right)$ since $y^{k} \in \mathcal{R}\left(x^{\prime}\right)$. Thus $g\left(x^{k}\right)+r I\left(x^{k}\right) \leq g\left(x^{\prime}\right)+r I\left(x^{\prime}\right)$, which is a contradiction.

If the rounding of $x^{k}$ is not unique, then it is possible that

$$
x^{k} \in \arg \min _{F_{L P}}\left\{g(\cdot)+r\left\|\cdot-y^{k}\right\|_{1}\right\}
$$

for some $y^{k} \in \mathcal{R}\left(x^{k}\right)$, but $x^{k}$ is not a local minimum of $g+r I$. In this case, arguments in Proposition 5.2 show that there must exist $\hat{y}^{k} \in \mathcal{R}\left(x^{k}\right)$ such that $x^{k} \notin \arg \min _{F_{L P}}\left\{g(\cdot)+r\left\|\cdot-\hat{y}^{k}\right\|_{1}\right\}$. We thus have the following result.

PROPOSITION 5.3. If for all $y \in \mathcal{R}\left(x^{k}\right), x^{k} \in \arg \min _{F_{L P}}\left\{g(\cdot)+r\|\cdot-y\|_{1}\right\}$, then $x^{k}$ is a local minimum of $g+r I$.

Proof. There exists a neighborhood of $x^{k}$, say, $N\left(x^{k}\right)$, such that for $x^{\prime} \in N\left(x^{k}\right)$, $\mathcal{R}\left(x^{\prime}\right) \subseteq \mathcal{R}\left(x^{k}\right)$. The result now follows from arguments in Proposition 5.2.

In general, it will not be easy to check the condition of Proposition 5.3, since the size of $\mathcal{R}\left(x^{k}\right)$ could be exponential in the number of variables. In practice, it is often the case that solutions to linear relaxations of binary integer programs have many variables at 0.5 . Thus we don't expect algorithms to search all possible roundings. However, one could view the minor perturbation step of the FP method, as described, for example, in [5], as attempting a randomized search of alternative roundings. The perturbation step selects a random number of variables (denoted by TT) to "flip," i.e., change the rounding direction, and chooses the variables with highest fractional value. Thus variables either at 0.5 in the LP solution, or near it, are chosen first to flip.

Thus we can view FP methods as seeking local minima of $g+r I$, first by using the discrete proximal point algorithm to find $x^{k} \in \arg \min _{F_{L P}}\left\{g(\cdot)+r\left\|\cdot-y^{k}\right\|_{1}\right\}$, 
and second, if the rounding $y^{k}$ is not unique, by randomly searching the set of all roundings. Of course, current FP methods do not explicitly choose the number of variables to flip in minor perturbation based on the number of variables at or near 0.5 in the current LP solution, so when the latter is small, the minor perturbation step tries to escape the nearby local minimum.

We are able to prove a converse form of Proposition 5.2- that if $x^{k}$ is a strict local minimum of $g+r I$, then the discrete proximal point algorithm cycles-when $g$ is convex. This result does not depend on a unique rounding.

Remark 5.4. The assumption that we have a strict local minimum of $g+r I$ at $x^{k}$ is easily satisfied. For any convex function $g$ take

$$
r>\max \left\{\|z\| \mid z \in \partial g\left(x^{k}\right)\right\} .
$$

Then if $x^{k}$ is a local minimum of $g+r I$, it is a strict local minimum.

Proposition 5.5. Suppose $g$ is convex and $x^{k}$ is a strict local minimum of $g+r I$ over $F_{L P}$. Then the discrete proximal point algorithm cycles at $x^{k}$.

Proof. Arguing by contradiction, assume $x^{k} \notin \arg \min _{F_{L P}}\left\{g(\cdot)+r\left\|\cdot-y^{k}\right\|_{1}\right\}$. Then we have

$$
g\left(x^{k+1}\right)+r\left\|x^{k+1}-y^{k}\right\|_{1} \leq g\left(x^{k}\right)+r\left\|x^{k}-y^{k}\right\|_{1} .
$$

Define

$$
x(\lambda):=(1-\lambda) x^{k}+\lambda x^{k+1}
$$

then $x(\lambda) \in F_{L P}$ for all $\lambda \in[0,1]$ and using the convexity of $g$ we have

$$
\begin{aligned}
g(x(\lambda))+r I(x(\lambda)) \leq & g(x(\lambda))+r\left\|x(\lambda)-y^{k}\right\|_{1} \\
\leq & (1-\lambda)\left[g\left(x^{k}\right)+r\left\|x^{k}-y^{k}\right\|_{1}\right] \\
& +\lambda\left[g\left(x^{k+1}\right)+r\left\|x^{k+1}-y^{k}\right\|_{1}\right] \\
\leq & (1-\lambda)\left[g\left(x^{k}\right)+r\left\|x^{k}-y^{k}\right\|_{1}\right] \\
& +\lambda\left[g\left(x^{k}\right)+r\left\|x^{k}-y^{k}\right\|_{1}\right] \\
= & g\left(x^{k}\right)+r\left\|x^{k}-y^{k}\right\|_{1}=g\left(x^{k}\right)+r I\left(x^{k}\right) .
\end{aligned}
$$

We can take $\lambda$ arbitrarily small so that $x(\lambda)$ can be in any small neighborhood of $x^{k}$. Thus $x^{k}$ cannot be a strict local minimum and so the result is proved by contradiction.

In summary we have shown that in the convex case, when $r$ is large enough, the discrete proximal point algorithm terminates only when a local minimum of $g+r I$ over $F_{L P}$ has been computed, and such local minima are exactly the points at which the algorithm cycles.

The use of penalties to produce a continuous version of an IP has been studied in [26] and [24]. In these papers, global minimizers of certain concave penalty functions are shown to correspond to solutions of an IP. We now compare our approach here with that of [26] and [24].

Proposition 5.6. Suppose $g$ is Lipschitz continuous and $n=m$ (we have a pure $I P)$. Then for $r>0$ sufficiently large, global minimizers of $g+r I$ over $F_{L P}$ coincide with the optimal solutions of the IP.

Proof. Let $\bar{x} \in \arg \min _{x \in F_{L P}}[g+r I]$. Then

$$
g(\bar{x})+r I(\bar{x}) \leq g(y)+r I(y)=g(y) \quad \text { for all } y \in F .
$$


Thus when $\bar{x}$ is integral we have $g(\bar{x})+r I(\bar{x})=g(\bar{x})$ and so $\bar{x}$ must be an optimal solution of the IP, establishing that global minimizers are optimal IP solutions. Thus we only need to establish that $\bar{x}$ must is integral when $r>0$ is sufficiently large.

Now there exists a fixed neighborhood $B_{\delta}(y)$ of each $y \in F$ within which $I(x)=$ $\|x-y\|_{1}$ for all $x \in B_{\delta}(y)$. Shrink $\delta$, if necessary, in order to ensure that $\left\{B_{\delta}(y) \mid\right.$ $y \in F\}$ is not a cover of $F_{L P}$. Consequently

$$
G:=F_{L P} \cap\left\{\bigcup_{y \in F} B_{\delta}(y)\right\}^{c}
$$

is a closed set containing no integral points and so $\min \{I(x) \mid x \in G\}:=\epsilon>0$. Counter to the assertion we assume that $\bar{x}(r) \in \arg \min _{x \in F_{L P}}[g+r I]$ is not integral for any sufficiently large $r>0$. Let $\eta(r):=\min _{y \in F}\|\bar{x}(r)-y\|_{1}=I(\bar{x}(r))$ and by assumption $\eta(r)>0$. A simple argument shows that $\eta(r)$ is a monotonically nonincreasing as a function of $r$. (Suppose $r^{\prime}>r$. By definition $g\left(\bar{x}\left(r^{\prime}\right)\right)+r^{\prime} I\left(\bar{x}\left(r^{\prime}\right)\right) \leq$ $g(\bar{x}(r))+r^{\prime} I(\bar{x}(r))$ and $g\left(\bar{x}\left(r^{\prime}\right)\right)+r I\left(\bar{x}\left(r^{\prime}\right)\right) \geq g(\bar{x}(r))+r I(\bar{x}(r))$. Subtracting gives $\left(r^{\prime}-r\right) I\left(\bar{x}\left(r^{\prime}\right)\right) \leq\left(r^{\prime}-r\right) I(\bar{x}(r))$.)

Let $r$ be sufficiently large so that $r>\max \left\{L, L \frac{\eta(r)}{\epsilon}\right\}:=\bar{r}$, where $L>0$ is the Lipschitz constant of $g$. With this fixed $r$ we will now drop reference to $r$ in $\bar{x}$ and $\eta$.

By definition $g(\bar{x})+r I(\bar{x}) \leq g(y)+r I(y)$ for all $y \in F$. Denote

$$
\begin{aligned}
\operatorname{lev}_{g+r I}(y) & :=\{x \mid g(x)+r I(x) \leq g(y)+r I(y)\} \\
& =\{x \mid g(x)-g(y)+r[I(x)-I(y)] \leq 0\} \\
& \subseteq\left\{x \mid \frac{I(x)-I(y)}{\|x-y\|_{1}} \leq \frac{1}{r} L\right\} \cup\{y\} .
\end{aligned}
$$

By definition $\bar{x} \in \operatorname{lev}_{g+r I}(y)$ and $y \in \operatorname{lev}_{g+r I}(y)$ for each $y \in F$. Using (5.2) and $r>L$ we see that $y$ is isolated point in the $\operatorname{set} \operatorname{lev}_{g+r I}(y)$ in that

$$
\{x \mid g(x)+r I(x) \leq g(y)+r I(y)\} \cap\left[B_{\delta}(y) \backslash\{y\}\right]=\emptyset .
$$

Thus $\|\bar{x}-y\|_{1} \geq \delta>0$ for all $y \in F$ implying $\bar{x} \in G$. Let $\bar{y} \in \arg \min _{y \in F}\|\bar{x}-y\|_{1}$ and by assumption $I(\bar{x})>0$. By $(5.1), \bar{x} \in G$, and the definition of $\bar{y}$ we have

$$
L \frac{\eta}{\epsilon}=L \frac{\|\bar{y}-\bar{x}\|_{1}}{\epsilon} \geq L \frac{\|\bar{y}-\bar{x}\|_{1}}{I(\bar{x})} \geq \frac{g(\bar{y})-g(\bar{x})}{I(\bar{x})} \geq r
$$

and so arrive at a contradiction. Thus $\bar{x} \in F$ is the optimal solution of the IP for $r$ sufficiently large.

This last result indicates that (major) random restarts in FP methods can be viewed as a way of attempting to find a global minimizer of $g+r I$. Similar techniques are used in global optimization.

Remark 5.7. In [26] and [24] various penalty functions for the integrality constraint are suggested. Suppose the function $\rho$ of section 3 is defined using the functional form

$$
\rho(x):=\sum_{i} p\left(\left|x_{i}\right|\right),
$$

where $p$ is one of the functions (3.2) to (3.5), all of which are integer compatible regularization functions. Then the induced penalty on integrality that would follow 
from a similar analysis to that made in this paper would be

$$
\rho(x)=\min _{y \mathcal{I} \in \mathbf{Z}^{m}} \sum_{i} p\left(\left|x_{i}-y_{i}\right|\right) .
$$

For binary problems we have for (3.4) and (3.5) that

$$
\begin{gathered}
\rho(x)=\sum_{i} \min \left\{1-\exp \left(-\alpha\left|x_{i}\right|\right), 1-\exp \left(-\alpha\left(\left|1-x_{i}\right|\right)\right)\right\}, \\
\rho(x)+\frac{n}{2}=\sum_{i} \min \left\{\left[1+\exp \left(-\alpha\left|x_{i}\right|\right)\right]^{-1},\left[1+\exp \left(-\alpha\left(\left|1-x_{i}\right|\right)\right)\right]^{-1}\right\}
\end{gathered}
$$

The only difference between these penalties and that given by (5.3) using (3.2) to (3.5) is that the parameter $\alpha$ directly penalizes the deviation of each $\left|x_{i}-y_{i}\right|$ from zero. In our approach we use an "external" multiplier $r$ to increase the penalty, while [24] use an "internal" multiplier $\alpha$. In order to reproduce the approach of [24] we would require a rewrite of the theory presented here to incorporate the use of this alternative penalty parameter $\alpha$ instead of the parameter $r$ into our framework. In $[24]$ the authors prove results similar to Proposition 5.6.

6. Incorporating cutting planes within FP. These results indicate that the points at which the discrete proximal point algorithm cycles are local minimizers of $g+r I$ over $F_{L P}$ (at least when $r$ is sufficiently large and $g$ convex). If we want to escape cycling episodes we need to change the structure of the optimization problem we are, in effect, solving. One way is to remove (or cut) that part of the region $F_{L P}$ that is associated with a nonintegral local minimum. This changes the feasible region over which we are minimizing. Cuts have a long history in integer programming and are used extensively in the branch and bound algorithm to help with fathoming of nodes. We have an entirely different motivation for suggesting them here.

Before giving details of our use of cutting planes, we first note some interesting connections with the work of Bonami et al. [8] on a feasibility pump approach to nonlinear MIP (NLMIP). In [8], the FP rounding step is replaced with mixed integer linear program (MILP) in which the linear constraints form an outer approximation to the original nonlinear constraints. In numerical experiments, this MILP is not necessarily solved to optimality but is partially solved until no improvement has been observed over a number of nodes exceeding a given limit. Two types of cuts are added to this MILP: (i) valid inequalities for the set defined by the nonlinear constraints (these give rise to the enhanced method described in [8]), and (ii) cutting planes generated by CPLEX in the course of its branch-and-cut (partial) solve. We note that a cut of type (i) can be generated at every iteration and that these are held over from one iteration of the method to the next, but there is no discussion of whether CPLEX cuts are retained for subsequent MILP solves. We also note that cuts of type (i) separate the integer "rounded" points from the feasible set defined by the nonlinear constraints, and so adding them to the problem solved in the "rounding step" prevents cycling. An alternative method to avoid some cycling in NLMIPs is used in [10], where a tabu list is kept of recently found solutions.

Our approach to the use of cutting planes is complementary. Rather than finding the FP iterate by solving

$$
x^{k+1} \in \arg \min _{x}\left\{c^{T} x+r\left\|y^{k}-x\right\|_{1}: A x \geq b, x \in \mathbf{R}^{n}\right\},
$$

Copyright $@$ ( ) by SIAM. Unauthorized reproduction of this article is prohibited. 
we instead attempt to solve the corresponding integer program

$$
x^{k+1} \in \arg \min _{x}\left\{c^{T} x+r\left\|y^{k}-x\right\|_{1}: A x \geq b, x_{\mathcal{I}} \in \mathbf{I}\right\}
$$

using a cutting plane algorithm. In other words, we seek to add cuts to the "projection step" rather than the rounding step. We terminate prematurely (i.e., before arriving at an integer solution) when the increase of the objective value in subsequent iterations of the cutting plane algorithm becomes small, indicating that additional cuts are of less value. Furthermore, we apply cutting conservatively, only seeking to add cuts when cycling is detected, rather than at every iteration. In the context of FP for problems with linear constraints, this approach has the benefit that the speed and ease of solution of the rounding step is retained, and the harder partial MIP solve is used only sparingly; otherwise a continuous linearly constrained problem is solved in the projection step.

The cutting process removes feasible LP points that are not in the convex hull of the integral feasible solutions, so we can (and do) retain prior cuts for use in subsequent iterations. As we wish to remove the LP feasible point that we are currently cycling around we must use the problem (6.2) in the cutting procedure.

Algorithm 1 outlines the basic FP framework given in [18] with the addition of the cutting phase given in step 1 . Starting with $x^{0}$ corresponding to the optimal solution over $F_{L P}$ and $y^{0}$ corresponding to its rounded value (denoted by $\left\lceil x^{0}\right\rfloor$ ), at each iteration the algorithm minimizes the distance between $y^{k}$ and $x^{k+1}$ during the pumping process (i.e., step 1 within the algorithm). Here, we solve the following LP that is clearly equivalent to (6.1) but parameterized differently:

$$
\begin{aligned}
\Delta_{\alpha}\left(x, y^{k}\right) & :=\sqrt{S} \min _{x \in F_{L P}}\left\{\alpha \frac{c^{T} x}{\|c\|_{2}}+(1-\alpha) \frac{\left\|x-y^{k}\right\|_{1}}{\sqrt{S}}\right\} \\
& \equiv \min _{x \in F_{L P}}\left\{\alpha \frac{\sqrt{S} c^{T} x}{\|c\|_{2}}+(1-\alpha)\left\|x-y^{k}\right\|_{1}\right\}
\end{aligned}
$$

for $\alpha \in[0,1]$, where $\|\cdot\|_{2}$ is the Euclidean norm and $S$ is the number of integer variables in the current problem. (This will be $l$ when the problem is a mixed binary problem.) One now drives $\alpha^{k} \rightarrow 0$ instead of $r^{k} \rightarrow \infty$. Note that the case $\alpha=0$ corresponds to the original FP of [17]. In practice, the OFP codes [1] use objective (6.3). This is also the objective used in the FP 2.0 of [18] and in all our numerical experiments.

The problem defined in (6.3) is formulated and solved as an LP [17]. For binary problems this reformulation does not require the introduction of any additional artificial variables within the LP. Thus, we can carry any cuts generated into future iterations. Unfortunately, for general integer programs, the addition of artificial variables changes the structure of the problem, making cuts in one iterate not directly transferrable to future iterates. Carrying an artificial variable forward would result in a rapid increase in the problem size so here we have focused on (mixed) binary problems.

As in standard implementations, Algorithm 1 performs a major restart when $Q$ consecutive attempts to recover from cycling within the inner loop fails. However, unlike standard FP, the inner loop contains both a perturbation and a cutting phase to recover from cycling. The cutting phase is selected with probability $1-\beta$, where $\beta \in[0,1]$ is a parameter of the algorithm. When $\beta=1$, the algorithm uses only the minor perturbations as per usual FP methods. 


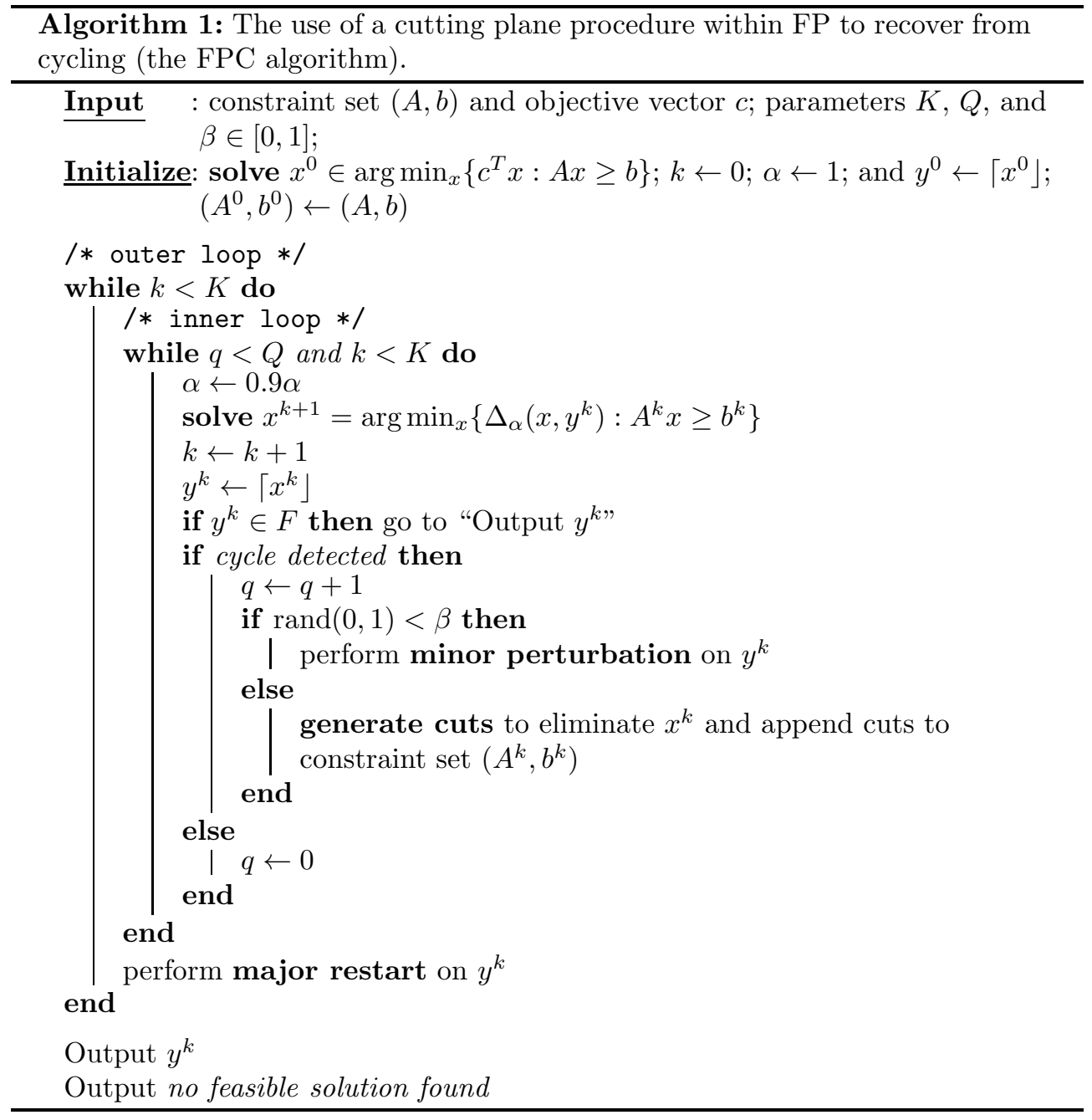

7. Computational results. The results reported in this section are based on 171 binary and mixed binary problems from MIPLIB2003 ${ }^{1}$ and $\mathrm{COR} @ \mathrm{~L}^{2}$ libraries for which the optimal solutions are known. The cutting plane procedure outlined in section 6 was implemented within the Feasibility Pump 2.0 [18] code that was provided by Fischetti and Salvagnin. In all cases, where applicable, we have used the default settings in FP 2.0 including all the parameters, tolerances, and limits related to FP 2.0.

To evaluate the merits of the proposed cutting plane procedure against FP 2.0, we have tested each of the 171 instances against both FP 2.0 and FP 2.0 augmented with the cutting plane procedure outlined in Algorithm 1, henceforth denoted FPC. We claim that any improvements in the solution quality from using cutting planes within the FP is due to the reduction in major restarts as a result of selectively tightening the LP feasible region enabling iterates to escape points where FP cycles, and not simply as a result of any tightening of the formulation around the LP optimal region.

\footnotetext{
${ }^{1}$ http://miplib.zib.de/miplib2003.php.

${ }^{2}$ http://coral.ie.lehigh.edu/data-sets/mixed-integer-instances.
} 
TABLE 7.1

Average number of major restarts for each scheme over all runs associated with instances belonging to each class.

\begin{tabular}{l|r|r|r|r}
\hline & FP 2.0 & FPP & FPC & FPPC \\
\hline Class A & 0.02 & 0.18 & 0.18 & 0.12 \\
Class B & 2.9 & 13.43 & 0.83 & 1.70 \\
Class C & 93.3 & 99.6 & 3.1 & 2.76 \\
\hline
\end{tabular}

To cement this claim, we also test all instances against a scheme denoted by FPP in which FP 2.0 is run on the root node relaxation of $F$ rather than on $F_{L P}$, i.e., FPP is the scheme in which FP 2.0 is run on the LP obtained by relaxing the integrality requirements but including any preprocessing and cuts that may be generated from the integrality requirements at the root node. Finally, we also test a scheme called FPPC in which FPC is combined with FPP, i.e., FPC is run on the root node relaxation of $F$ rather than on $F_{L P}$.

Note that in our computational experiments, we have used the available constraint propagation engine together with rounding rather than rounding only [18]. The constraint propagation complements our cutting framework as it can take advantage of the cuts generated. We note that when constraint propagation was turned off, sufficient improvement using cutting was obtained to make FPC without propagation at least as good as FP 2.0 with propagation. Finally, we found that $\beta=0.5$ gave an effective mechanism to recover from cycling, alternating randomly between minor perturbations and the cutting procedure. Experiments suggests that regular use of the cutting phase helps shape the feasible region more uniformly in a way that helps FPC to progress by avoiding restarts.

We use CPLEX $12.1^{3}$ to solve the LP (6.3), to solve the root node relaxation within FPP and FPPC, and to generate cuts within FPC. Since we cannot interject in the cut generation process of CPLEX, we have restricted our test bed to binary and mixed binary problems where artificial variables are not needed to solve (6.3), and thus the cuts generated can be passed to future iterates. (See the discussion in section 6.) In the case of FPC, the cut generation process is terminated when it fails to increase the objective value of (6.3) by at least $30 \%$ from the previous value. This occurs fairly rapidly and encourages progress without excessive time being consumed in the cutting phase.

Finally, due to the random effects introduced by perturbations and major restarts, each instance is tested on each of the 4 schemes on 10 independent runs. Furthermore, since the 171 instances present problems that pose varied levels of difficulty for FP 2.0, we classify each of the 171 instances into one of three classes depending on the number of major restarts needed before an integer feasible solution is obtained using standard FP 2.0. Class A includes 110 problems that terminated with no major restarts for at least 7 of the 10 runs, class B contains 30 problems not in class A with less than 10 major restarts for at least 7 out of 10 runs, and class $\mathrm{C}$ contains the remaining 31 problems.

Table 7.1 reports the average number of major restarts for each scheme over all runs associated with instances belonging to a given class. Hence, if $I_{A}, I_{B}$, and $I_{C}$ are the set of instances belonging to class A, B, and C, respectively, $S=$ $\{\mathrm{FP} 2.0$, FPP, FPC, FPPC $\}$ is the set of schemes, and $R_{i j}^{s}$ is the number of major restarts observed during the $j$ th run (out of 10) of instance $i$ using some scheme $s \in S$,

\footnotetext{
${ }^{3} \mathrm{http}: / /$ www-01.ibm.com/software/integration/optimization/cplex-optimizer.
} 
Class A

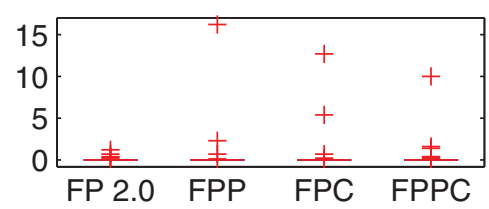

Class B

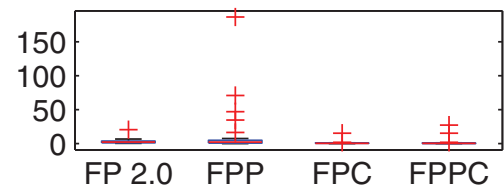

Class C

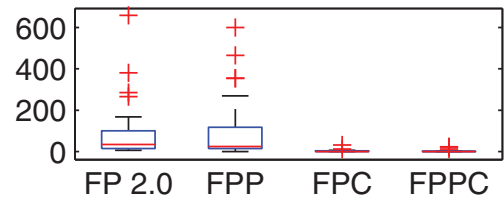

Class A (without outliers)

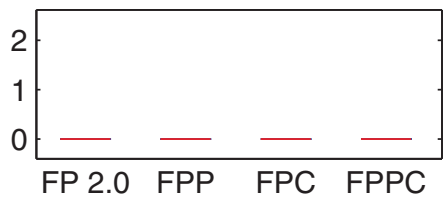

Class B (without outliers)

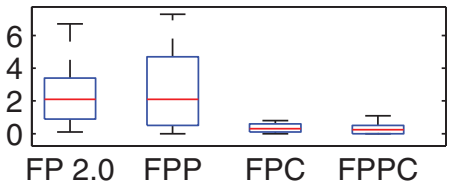

Class C (without outliers)

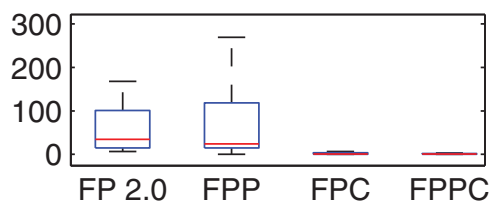

Number of Outliers

\begin{tabular}{c|r|rrrr}
\multicolumn{7}{|c}{ Number of Outliers } \\
\hline Class & No. Instances & FP 2.0 & FPP & FPC & FPPC \\
\hline A & 110 & 5 & 8 & 9 & 7 \\
B & 30 & 1 & 5 & 2 & 3 \\
C & 31 & 4 & 5 & 4 & 3 \\
\hline Total & 171 & 9 & 18 & 15 & 13 \\
\hline
\end{tabular}

FIG. 7.1. Distribution of the average number of major restarts by scheme and problem class, with and without outliers, and breakdown of the number of outliers for each scheme and class.

then for each scheme $s \in S$ and class $l \in\{A, B, C\}$, Table 7.1 reports

$$
\bar{R}_{l}^{s}=\frac{1}{10\left|I_{l}\right|} \sum_{i \in I_{l}} \sum_{j=1}^{10} R_{i j}^{s} .
$$

Additionally, the box plots given in Figure 7.1 give the distribution of the average (over the 10 independent runs for each instance) number of major restarts by scheme and problem class. More precisely, for each class $l \in\{A, B, C\}$, and each scheme $s \in S$, Figure 7.1 plots the distribution of values

$$
\left\{\frac{1}{10} \sum_{j=1}^{10} R_{i j}^{s}: i \in I_{l}\right\} .
$$

To present a clearer picture, Figure 7.1 includes box plots of these distributions with and without outliers. Values are considered as outliers if they are larger than $q_{3}+$ $1.5\left(q_{3}-q_{1}\right)$ or smaller than $q_{1}-1.5\left(q_{3}-q_{1}\right)$, where $q_{1}$ and $q_{3}$ are the 25 th and 75 th percentiles, respectively. The number of outliers for each scheme in each problem class is shown in the table at the bottom of Figure 7.1.

From Table 7.1 and Figure 7.1 it is clear that adding cuts strategically at points where FP cycles has a dramatic effect on reducing the number of major restarts for 


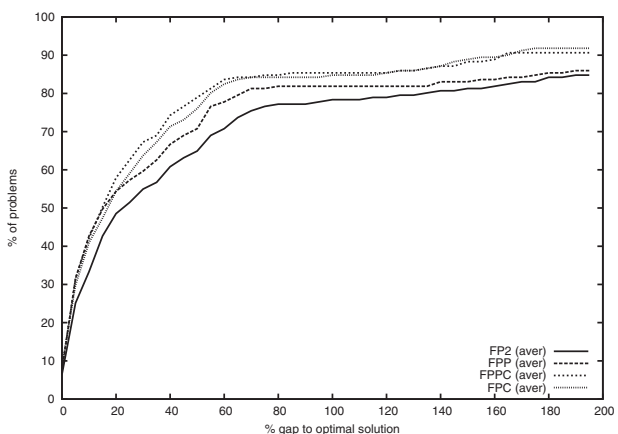

(a) All problems

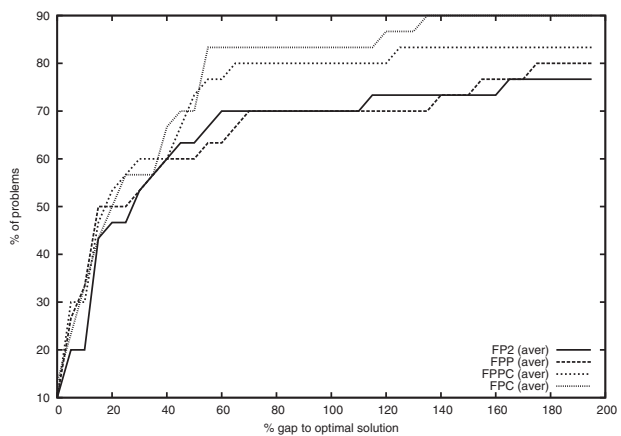

(c) Class B

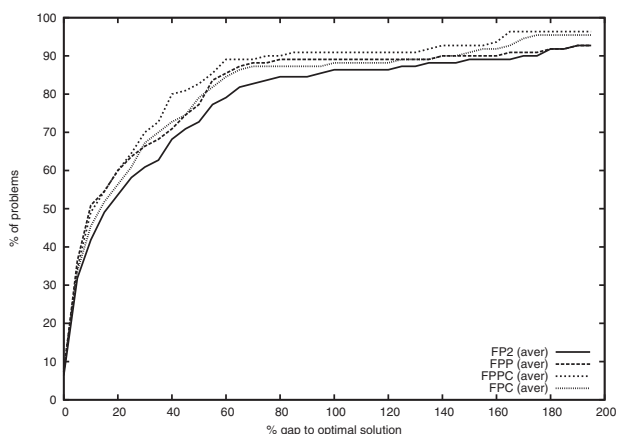

(b) Class A

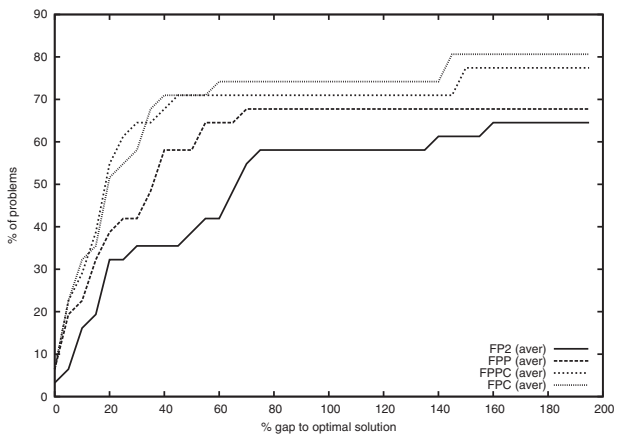

(d) Class C

FIG. 7.2. Percentage of instances where the average value of solutions obtained over the 10 independent runs by a given scheme is within a certain percentage to the optimal solution.

problems in classes B and C. FPP, FPC, and FPPC all increase the average number of major restarts for problems in class A. However, as can be observed from Figure 7.1, this is mainly due to a handful of outliers. For problems in class B, FPC reduces the number of major restarts by more than a factor of three on average; however, both FPP and FPPC have more restarts than FP 2.0 and FPC, respectively. This is not completely surprising. Indeed, tightening the formulation through cuts at the root node is likely to make the relaxation more fractional. The binary knapsack problem is a classic example of this. Here, the LP relaxation has only one fractional variable, whereas any strengthening of the problem with the addition of cuts is likely to introduce greater fractionality. Similarly, for problems in class C, FPC reduces the number of major restarts by more than a factor of 30 over FP 2.0 on average, whereas FPPC and FPP are comparable to FPC and FP 2.0, respectively. Finally, it is worth noting that although the number of major restarts is reduced significantly, the overall number of FP iterations remains comparatively unchanged within the various schemes. We next present the main results demonstrating the impact of the reduction in major restarts on solution quality and thus the efficacy of the proposed cutting scheme.

Figures 7.2 and 7.3 provide performance profiles for the average and best solutions obtained respectively by the various schemes and for the various class of problems. Each plot gives the percentage of instances where a solution was obtained by a given scheme within a certain percentage to the optimal solution. More precisely, if $J_{i}^{s} \subseteq$ $\{1, \ldots, 10\}$ is the subset of runs of scheme $s$, for instance, $i$, that produced a solution, 


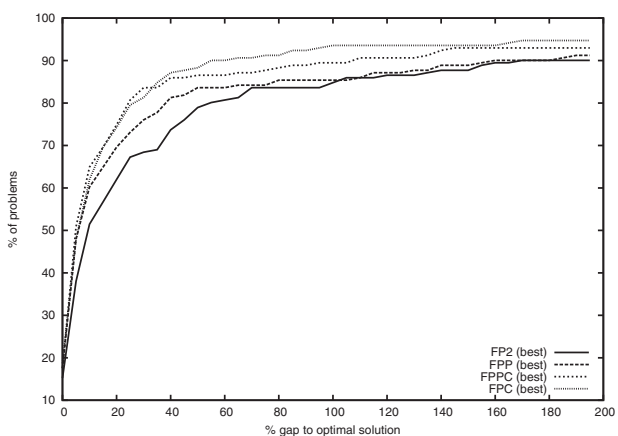

(a) All problems

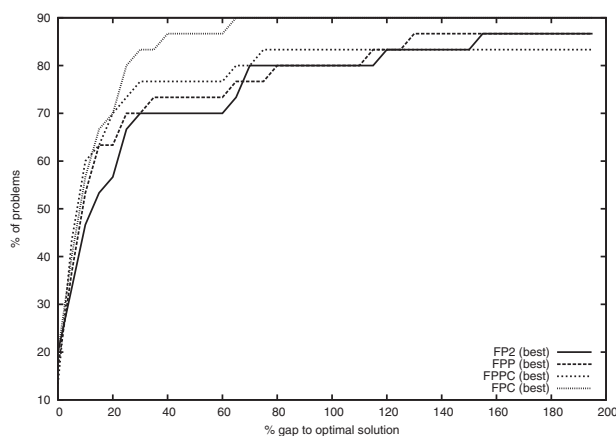

(c) Class B

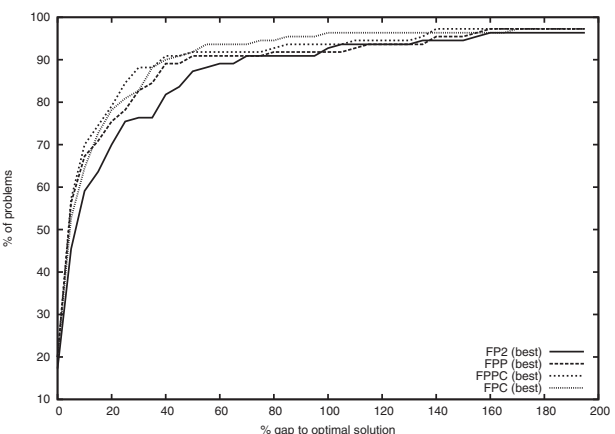

(b) Class A

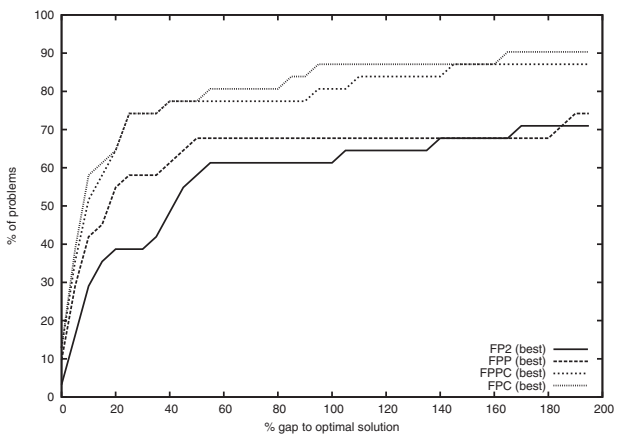

(d) Class C

FIG. 7.3. Percentage of instances where the value of the best solution obtained over the 10 independent runs by a given scheme is within a certain percentage to the optimal solution.

and $Z_{i j}^{s}$ is the objective value of the best solution found, for instance, $i$, during run $j \in J_{i}^{s}$, then we define

$$
\bar{Z}_{i}^{s}=\frac{1}{\left|J_{i}^{s}\right|} \sum_{j \in J_{i}^{s}} Z_{i j}^{s}
$$

to be the average objective value of solutions found, for instance, $i$, using scheme $s$, and

$$
\hat{Z}_{i}^{s}=\max _{j \in J_{i}^{s}} Z_{i j}^{s}
$$

to be the best objective value among solutions found for, instance, $i$ using scheme $s$. We set $\bar{Z}_{i}^{s}$ and $\hat{Z}_{i}^{s}$ to $\infty$ if $J_{i}^{s}=\{\emptyset\}$. Moreover, given $Z_{i}^{*}$, the optimal solution, for instance, $i$, the percentage gap to the average objective value of solutions found, for instance, $i$ using scheme $s$, is given by

$$
\operatorname{avgGap} s=\frac{\left(\bar{Z}_{i}^{s}-Z_{i}^{*}\right)}{\max \left\{\left|Z_{i}^{*}\right|, 1\right\}} \times 100,
$$

and similarly, the percentage gap to the best solution found, for instance, $i$ using scheme $s$, is given by

$$
\operatorname{bestGap}_{i}^{s}=\frac{\left(\hat{Z}_{i}^{s}-Z_{i}^{*}\right)}{\max \left\{\left|Z_{i}^{*}\right|, 1\right\}} \times 100 .
$$

Copyright (c) by SIAM. Unauthorized reproduction of this article is prohibited. 

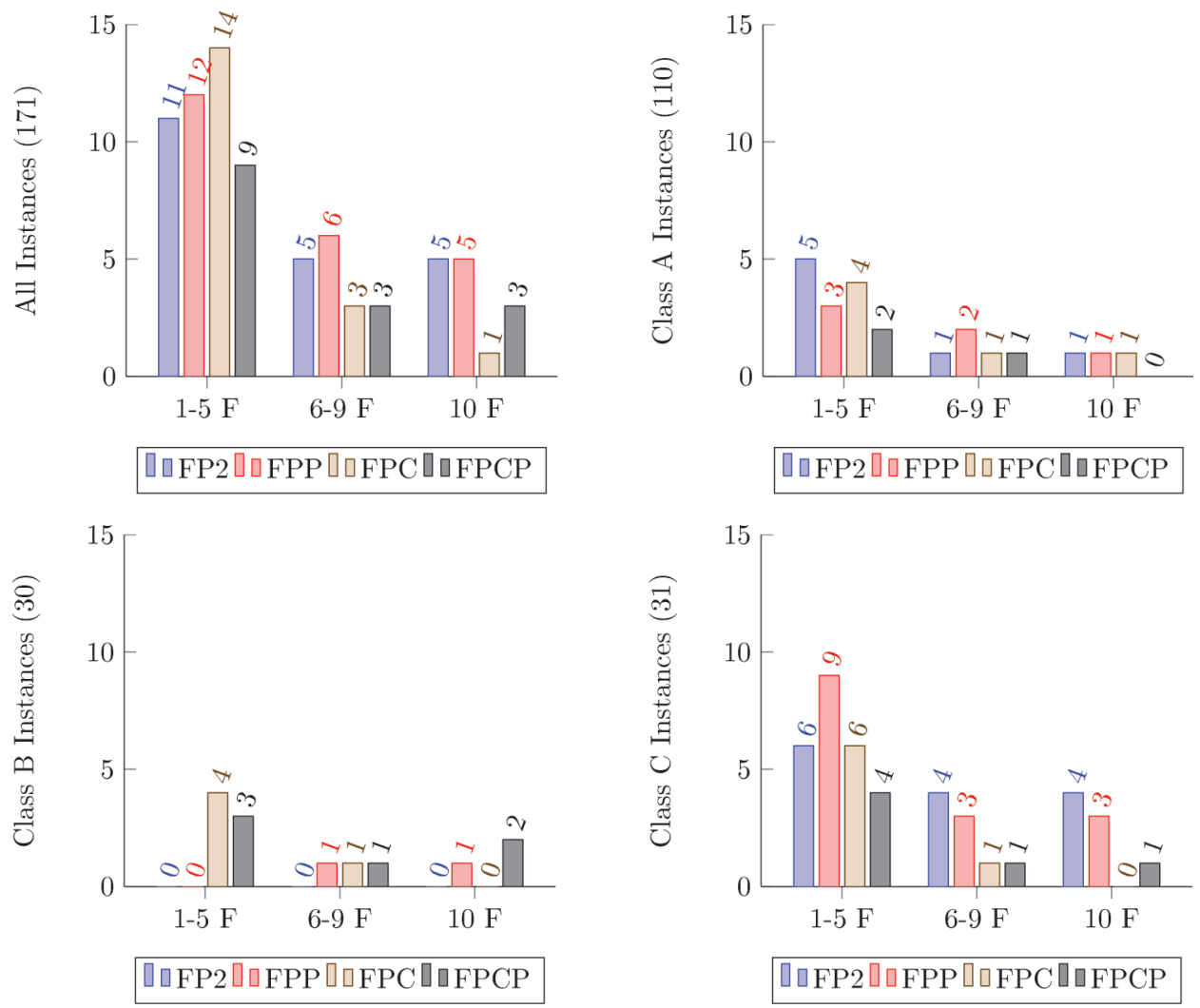

FIG. 7.4. Number of instances in each class with 1-5 failures, 6-9 failures, and 10 failures for each scheme. Out of 1710 runs, FP2 failed to find a feasible point 113 times, FPP 119 times, FPC 58 times, and FPCP 68 times. FP2 fails to find a feasible solution in all 10 runs for an instance 5 times, while this occurs for FPC only once.

Then, for each percentage $p \in[0,100]$ and scheme $s$, Figure 7.2 plots the number of instances whose average objective is within $p$ percent of the optimal solution, i.e., plots $p$ against the number of instances $i$ that have avgGapis $\leq p$. Similarly, Figure 7.3 plots the number of instances $i$ whose best objective is within $p$ percent of the optimal solution, i.e., plots the number of instances $i$ where bestGap ${ }_{i}^{s} \leq p$. Figures 7.2(a) and 7.3(a) present this information over all instances for each of the four schemes, while Figures 7.2(b)-7.2(d) and 7.3(b)-7.3(d) present this information for problems in each of the three classes $\mathrm{A}, \mathrm{B}$, and $\mathrm{C}$, respectively.

Since average gap can be reported only over runs which found a solution, it is possible that finding no solution appears better under this statistic than finding a poor-quality solution. To check that our conclusions are not being influenced by such a distortion, we report statistics for the incidence of failure to find a feasible solution for each scheme in Figure 7.4.

Although there is not much that separates FPC and FPPC, from Figures 7.2(a) and 7.3(a) it is clear that on the whole, the cutting plane approaches outperform FP 2.0 and FPP. When examining the three classes in isolation, we observe that for problems in class A, it is the tightening of the feasible region (FPP) that yields most of the improvement. This is to be expected as an attempt to reduce major restarts (FPC, FPPC) cannot be very useful for problems that do not require many major 
TABLE 7.2

Average time (in seconds) taken by each scheme over all runs associated with instances belonging to given class.

\begin{tabular}{l|r|r|r|r}
\hline & FP 2.0 & FPP & FPC & FPPC \\
\hline Class A & 24.23 & 43.91 & 32.11 & 49.72 \\
Class B & 13.07 & 21.60 & 81.58 & 81.73 \\
Class C & 18.52 & 23.65 & 63.46 & 69.38 \\
\hline
\end{tabular}

restarts to begin with. Here FPC does worse than FPP but better than FP 2.0, while FPPC does better than FPP. The true impact of the proposed cutting scheme as a mechanism for recovering from cycling is evident for the harder problems within classes B and C. Indeed, the percentage of problems that could be solved to within $10 \%$ of optimality is almost twice that in FPC and FPPC as compared to FP 2.0 for class $\mathrm{C}$, and almost $50 \%$ more for problems in class B.

Admittedly, by examining the relative performance of FPP versus FP 2.0, we can conclude that some of the aforementioned gains can be attributed to simply tightening the formulation at the root node. However, there are significant gains to be had over FPP by using cutting planes strategically to avoid cycling. When comparing the average objective values obtained over the 10 independent runs, FPC is better on 21 occasions and worse on only 3 occasions for the 30 instances belonging to class $\mathrm{B}$, i.e., $\left|\left\{i \in I_{B}: \bar{Z}_{i}^{F P C}<\bar{Z}_{i}^{F P 2.0}\right\}\right|=21$ and $\left|\left\{i \in I_{B}: \bar{Z}_{i}^{F P C}>\bar{Z}_{i}^{F P 2.0}\right\}\right|=3$. For the same measure, FPC is better on 24 occasions and worse on only 6 occasions for the 31 instances in class C. Moreover, when comparing the best objective value found over the 10 independent runs for instances in class C, FPC was better than FP 2.0 on 26 occasions and worse on only 4 occasions, i.e., $\left|\left\{i \in I_{C}: \hat{Z}_{i}^{F P C}<\hat{Z}_{i}^{F P 2.0}\right\}\right|=26$ and $\left|\left\{i \in I_{C}: \hat{Z}_{i}^{F P C}>\hat{Z}_{i}^{F P 2.0}\right\}\right|=4$. Even if we compare the best solution found by FP 2.0 over the 10 independent runs with the average solution found by FPC, the comparison still looks favorable for FPC, yielding 18 wins and 12 losses, i.e., $\left|\left\{i \in I_{C}: \bar{Z}_{i}^{F P C}<\hat{Z}_{i}^{F P 2.0}\right\}\right|=18$ and $\left|\left\{i \in I_{C}: \bar{Z}_{i}^{F P C}>\hat{Z}_{i}^{F P 2.0}\right\}\right|=12$.

Finally, Figure 7.4 confirms that the average gap performance is not distorted by failure to find a feasible solution: the schemes with cutting fail to find a feasible solution less often than those without and fail less seriously in the sense that there is a lower incidence of a high proportion of failures for an instance. For example, FPC fails to find a solution in all 10 runs only once, while FP2 fails 5 times. Of the total 1710 runs FP2 fails 113 times and FPC 58 times. See Appendix B and the tables therein, which provide detailed statistics comparing the results of FP 2.0 with FPC for individual instances.

Of course, the gains in terms of solution quality obtained from using cuts to tighten the formulation and/or recover from cycling come at a cost-in particular, the additional computational effort required in cut generation. Table 7.2 reports the average time (in seconds) for each scheme over all runs associated with instances belonging to a given class. Hence, if $T_{i j}^{s}$ is the time taken for the $j$ th run (out of 10) of instance $i$ using some scheme $s \in S$, then for each scheme $s \in S$ and class $l \in\{A, B, C\}$, Table 7.2 reports

$$
\bar{T}_{l}^{s}=\frac{1}{10\left|I_{l}\right|} \sum_{i \in I_{l}} \sum_{j=1}^{10} T_{i j}^{s} .
$$

The box plots given in Figure 7.5 give the distribution of the average time (over the 10 independent runs for each instance) taken by each scheme for problems in each 
Class A

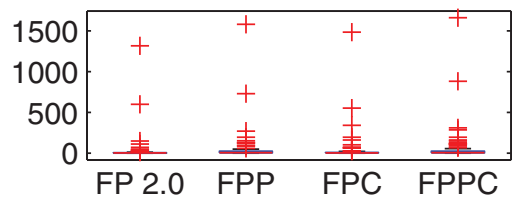

Class B

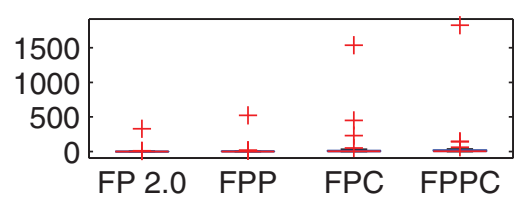

Class C

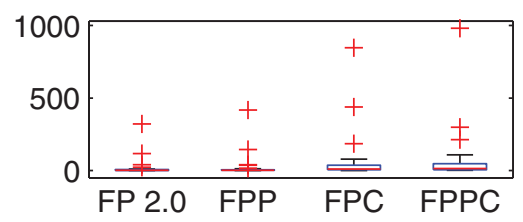

Class A (without outliers)

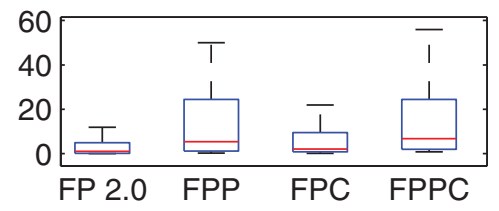

Class B (without outliers)

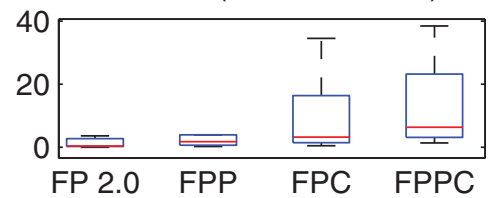

Class C (without outliers)

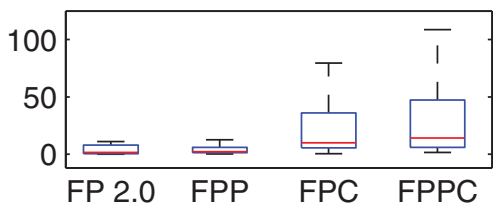

FIG. 7.5. Distribution of the average time taken (in seconds) by each scheme for problems in each class.

class. More precisely, for each class $l \in\{A, B, C\}$ and each scheme $s \in S$, Figure 7.1 plots the distribution of points

$$
\left\{\frac{1}{10} \sum_{j=1}^{10} T_{i j}^{s}: i \in I_{l}\right\} .
$$

As before, Figure 7.5 includes box plots with and without outliers.

From Table 7.2 and Figure 7.5 it is clear that incorporating cut generation within FP places a heavy burden on the computational effort required. However, almost all of the reported increase in time is due to the cut generation process itself. Hence, we are optimistic that through further tuning and a proper software solution and integration of the cutting procedure with FP (as we are using CPLEX as a "black box" only), any additional computational burden is likely to pay dividends in practical situations where time may also be of the essence.

8. Further directions. The connection of FP methods with proximal point algorithms, and the theoretical results we have presented here, motivate several new directions of exploration for future research. One of our key insights is that the FP pumping cycle appears to be seeking local minima of a weighted combination of the original objective function and a measure of integrality. The method cycles at LP feasible points which are either local minima or have multiple alternate roundings. Alternate roundings could break the cycle; if this is not possible, then the point must be a local minimum. This insight motivates a different approach to the FP minor perturbation step, which chooses at random a number of variables to have their rounding changed and changes those variables with fractional values closest to 0.5 . Since variables at 0.5 are precisely those causing alternative roundings, it thus 
seems that minor perturbation has two functions: (a) to carry out a randomized search of the set of alternative roundings when these are not unique, to check whether the algorithm is likely to be at a local minimum, and (b) to escape the local minimum. If the number of variables to have their rounding changed exceeds the number at or near 0.5 , then (b) is occurring; otherwise it is (a). Since the minor perturbation step does not look at the number of variables at or near 0.5 , there is an opportunity to redesign this step: do (a) until there is convincing evidence the current point is a local minimum, and then (b) escape it. It could be that current minor perturbation does (a) quite well and our cutting approach does (b) well, but in both standard FP methods and our cutting plane modification, steps (a) and (b) occur in a mixed sequence.

In our cutting plane approach we have focused on the projection step. It would also be interesting to test the trade-off between the time needed for the rounding step and the resulting solution quality with an approach such as that of Bonami et al. [8]. FP2.0 [18] goes some of that way, incorporating information from the original problem constraints in the rounding step by propagation. But the addition of a limited number of cutting planes to form a MIP in place of the rounding step could be attractive if the MIP solve is fast enough.

Another direction of further research is to explore the role of the weighting parameter, which weights integrality versus the original objectives. Current FP methods simply increase this parameter to increasingly emphasize integrality. However, the theoretical results show that optimal solutions require the parameter to be sufficiently small. It seems likely that further experimentation with how this parameter is adjusted could yield more effective algorithms, and in particular that phases in which the parameter is also decreased may be beneficial to producing higher-quality solutions.

\section{Appendix A. Proof of Proposition 3.7.}

Proof. In view of Lemma 3.5, part 3, we need only show that if $x=y_{0}$ is the unique solution of (3.8) for $y=y_{0}$, then it is a strict local minimum of $\varphi_{r}$. Note that we already know that $y_{0}$ is integral. Consider $K:=\varphi_{r}\left(y_{0}\right)+2$ and denote

$$
F_{K}^{\mathbf{I}}\left(y_{0}\right):=\left\{x_{\mathcal{I}} \in \mathbf{I} \mid \inf _{x_{\mathcal{I}} \in \mathbf{R}^{n-m}}\left\{f\left(x_{\mathcal{I}}, x_{\mathcal{R}}\right)+r \rho\left(\left(x_{\mathcal{I}}, x_{\mathcal{R}}\right)-y_{0}\right)\right\} \leq K\right\},
$$

which is bounded due to the fact that $F_{K}\left(y_{0}\right)$ as defined in (3.10) is bounded. Now $y \mapsto \varphi_{r}(y)$ is upper semicontinuous when $y \mapsto \rho(x-y)$ is continuous. Thus $y$ close to $y_{0}$ we have $\varphi_{r}(y)+1<\varphi_{r}\left(y_{0}\right)+2=K$. Consequently for $y$ sufficiently close to $y_{0}$ we have $F_{K-1}^{\mathbf{I}}(y) \subseteq F_{K}^{\mathbf{I}}\left(y_{0}\right)$. Using the discreteness of the integral part of the solutions in $F_{K}^{\mathbf{I}}\left(y_{0}\right)$ and that $y_{0}$ is the unique minimum, we have some $\varepsilon>0$ such that

$$
\begin{aligned}
f(x)+r \rho\left(x-y_{0}\right) & \geq \inf _{x_{\mathcal{R}} \in \mathbf{R}^{n-m}}\left\{f\left(x_{\mathcal{I}}, x_{\mathcal{R}}\right)+r \rho\left(\left(x_{\mathcal{I}}, x_{\mathcal{R}}\right)-y_{0}\right)\right\} \\
& \geq f\left(y_{0}\right)+2 \varepsilon=\varphi_{r}\left(y_{0}\right)+2 \varepsilon \text { for all } x_{\mathcal{I}} \\
& \neq\left(y_{0}\right)_{\mathcal{I}} \text { with } x_{\mathcal{I}} \in F_{K}^{\mathbf{I}}\left(y_{0}\right) .
\end{aligned}
$$

By lower semicontinuity of $\rho$ and compactness of $F_{K}$, for $y$ sufficiently close to $y_{0}$ and all $x$ with $x \in F_{K}\left(y_{0}\right)$ we have

$$
r \rho(x-y) \geq r \rho\left(x-y_{0}\right)-\varepsilon .
$$


Consequently for all $y$ sufficiently close to $y_{0}$

$$
f(x)+r \rho(x-y) \geq \varphi_{r}\left(y_{0}\right)+\varepsilon \quad \text { for all } x_{\mathcal{I}} \neq\left(y_{0}\right)_{\mathcal{I}} \text { with } x_{\mathcal{I}} \in F_{K}^{\mathbf{I}}\left(y_{0}\right) .
$$

Thus $\min _{x}\left\{f(x)+r \rho(x-y) \mid\left(y_{0}\right)_{\mathcal{I}} \neq x_{\mathcal{I}}\right.$ with $\left.x_{\mathcal{I}} \in F_{K}^{\mathbf{I}}\left(y_{0}\right)\right\} \geq \varphi_{r}\left(y_{0}\right)+\varepsilon$ for all $y$ close to $y_{0}$. Using $F_{K-1}^{\mathbf{I}}(y) \subseteq F_{K}^{\mathbf{I}}\left(y_{0}\right)$ for $y$ sufficiently close to $y_{0}$ we have

$$
\begin{aligned}
& \varphi_{r}(y) \\
& =\min \left\{\min _{x: x_{\mathcal{I}} \neq\left(y_{0}\right)_{\mathcal{I}}}\left\{f(x)+r \rho(x-y) \mid x_{\mathcal{I}} \in F_{K-1}^{\mathbf{I}}(y)\right\},\right. \\
& \left.\geq \min _{x: x_{\mathcal{I}}=\left(y_{0}\right)_{\mathcal{I}}}\left\{f(x)+r \rho(x-y) \mid x_{\mathcal{I}} \in F_{K-1}^{\mathbf{I}}(y)\right\}\right\} \\
& \geq \min \left\{\min _{x: x_{\mathcal{I}} \neq\left(y_{0}\right)_{\mathcal{I}}}\left\{f(x)+r \rho(x-y) \mid x_{\mathcal{I}} \in F_{K-1}^{\mathbf{I}}(y)\right\},\right. \\
& \left.\min _{x_{\mathcal{R}}}\left\{f\left(\left(y_{0}\right)_{\mathcal{I}}, x_{\mathcal{R}}\right)+r \rho\left(x_{\mathcal{R}}-y_{\mathcal{R}}\right)+r \rho\left(\left(y_{0}\right)_{\mathcal{I}}-y_{\mathcal{I}}\right)\right\}\right\} \\
& \geq \min \left\{\varphi_{r}\left(y_{0}\right)+\varepsilon, G_{r}\left(y_{\mathcal{R}}\right)+r \rho\left(\left(y_{0}\right)_{\mathcal{I}}-y_{\mathcal{I}}\right)\right\} .
\end{aligned}
$$

Now note that

$$
\begin{aligned}
G_{r}\left(\left(y_{0}\right)_{\mathcal{R}}\right) & \geq \varphi_{r}\left(y_{0}\right)=f\left(y_{0}\right) \\
& \geq \min _{z_{\mathcal{R}} \in \mathbf{R}^{n-m}}\left\{f\left(\left(y_{0}\right)_{\mathcal{I}}, z_{\mathcal{R}}\right)+r \rho\left(\left(\left(y_{0}\right)_{\mathcal{I}}, z_{\mathcal{R}}\right)-y_{0}\right)\right\}=G_{r}\left(\left(y_{0}\right)_{\mathcal{R}}\right)
\end{aligned}
$$

so $G_{r}\left(\left(y_{0}\right)_{\mathcal{R}}\right)=\varphi_{r}\left(y_{0}\right)$. Also $\left(y_{0}\right)_{\mathcal{R}}$ is a local minimum of $G_{r}$ because $G_{r}\left(y_{\mathcal{R}}\right) \geq$ $\varphi_{r}\left(\left(y_{0}\right)_{\mathcal{I}}, y_{\mathcal{R}}\right)$ for all $y$ and $y_{0}$ is a local minimum of $\varphi_{r}$, i.e., for $y_{\mathcal{R}}$ close to $\left(y_{0}\right)_{\mathcal{R}}$

$$
G_{r}\left(y_{\mathcal{R}}\right) \geq \varphi_{r}\left(\left(y_{0}\right)_{\mathcal{I}}, y_{\mathcal{R}}\right) \geq \varphi_{r}\left(y_{0}\right)=G_{r}\left(\left(y_{0}\right)_{\mathcal{R}}\right) .
$$

Thus for $y$ sufficiently close to $y_{0}$ we have $\varphi_{y}(y) \geq \varphi_{r}\left(y_{0}\right)$.

When we have an IP, then $G_{r}\left(y_{\mathcal{R}}\right)=\varphi_{r}\left(y_{0}\right)$ and so we have

$$
\varphi_{r}(y) \geq \min \left\{\varphi_{r}\left(y_{0}\right)+\varepsilon, \varphi_{r}\left(y_{0}\right)+r \rho\left(y_{0}-y\right)\right\} .
$$

Thus we have a strict local minimum at $y_{0}$. Furthermore, in the more general case of a MIP when $\left(y_{0}\right)_{\mathcal{R}}$ is a strict local minimum of $G_{r}$ then the inequality in (A.2) again implies $y_{0}$ must also be a strict local minimum of $\varphi_{r}$.

Appendix B. Detailed results for FP 2.0 and FPC. In Tables B.1, B.2, and B.3 we report detailed statistics related to the runs associated with FP 2.0 and FPC for each of the 171 instances. The first column gives the problem's name, and for each of the two schemes FP 2.0 and FPC, the remaining columns give the average gap to the optimal solution over the 10 independent runs (i.e., $\operatorname{avgGap}_{i}^{F P 2.0}$ and $\operatorname{avg} G a p_{i}^{F P C}$ ), the gap to the optimal solution from the best solution obtained over the 10 independent runs (i.e., bestGap $p_{i}^{F P 2.0}$ and bestGapi ${ }_{i}^{F C}$ ), the average number of FP iterations, the average number of major restarts (i.e., $\bar{R}_{i}^{F P 2.0}$ and $\bar{R}_{i}^{F P C}$ ), the average number of cuts generated (only for FPC), the average time taken (in seconds) (i.e., $\bar{T}_{i}^{F P 2.0}$ and $\bar{T}_{i}^{F P C}$ ), and the number of occasions (of the 10 independent runs) that a feasible solution was found. 
TABLE B. 1

Detailed statistics for FP2.0 and FPC for problems in class $A$.

\begin{tabular}{|c|c|}
\hline Name & \\
\hline & Ave \\
\hline & FP 2.0 \\
\hline aflow30a & 133.2 \\
\hline aflow $40 \mathrm{~b}$ & 41.5 \\
\hline air04 & 1.4 \\
\hline air05 & 2.8 \\
\hline cap6000 & 2.3 \\
\hline disktom & 0.0 \\
\hline & 969.2 \\
\hline fast 0507 & 5.0 \\
\hline fiber & 244.3 \\
\hline fixnet 6 & 12.2 \\
\hline harp2 & 9.3 \\
\hline $\operatorname{misc07}$ & 36.6 \\
\hline $\mathrm{mkc}$ & 49.8 \\
\hline $\bmod 011$ & 16.5 \\
\hline net12 & 41.2 \\
\hline nsrand-ipx & 60.9 \\
\hline nw04 & 31.2 \\
\hline p2756 & 123.4 \\
\hline pp08a & 63.9 \\
\hline pp08aCUTS & 38.6 \\
\hline protfold & 54.5 \\
\hline qiu & 500.5 \\
\hline seymour & 3.6 \\
\hline sp97ar & 21.3 \\
\hline $\mathrm{t} 1717$ & 149.3 \\
\hline bc1 & 36.4 \\
\hline binkar10-1 & 6.5 \\
\hline dano3-3 & 0.0 \\
\hline dano3-5 & 0.0 \\
\hline leo1 & 34.0 \\
\hline leo2 & 28.9 \\
\hline neos 1 & 39.5 \\
\hline neos5 & 5.0 \\
\hline neos 9 & 0.5 \\
\hline neos11 & 0.0 \\
\hline neos 12 & 16.9 \\
\hline neos 13 & 14.7 \\
\hline neos14 & 46.0 \\
\hline neos 15 & 52.0 \\
\hline neos- 476283 & 0.1 \\
\hline neos- 480878 & 2.4 \\
\hline neos- 495307 & 5.7 \\
\hline neos-503737 & 50.2 \\
\hline neos- 504674 & 77.4 \\
\hline neos-504815 & 68.4 \\
\hline neos-506428 & 176.0 \\
\hline neos-512201 & 62.9 \\
\hline neos-520729 & 0.3 \\
\hline neos- 525149 & 2.6 \\
\hline neos- 538867 & 179.1 \\
\hline neos-538916 & 278.1 \\
\hline neos-544324 & 12.0 \\
\hline neos-547911 & 9.2 \\
\hline neos-555694 & 24.0 \\
\hline neos- 565815 & 0.0 \\
\hline neos- 570431 & 14.4 \\
\hline neos-584146 & 0.0 \\
\hline neos- 584851 & 29.1 \\
\hline neos- 611135 & 187.8 \\
\hline neos- 611838 & 0.7 \\
\hline neos- 612125 & 0.5 \\
\hline neos- 612143 & 0.7 \\
\hline neos- 612162 & 0.7 \\
\hline neos- 631784 & 8.8 \\
\hline neos- 691058 & 56.6 \\
\hline neos- 691073 & 21.3 \\
\hline neos-738098 & 0.0 \\
\hline neos- 787933 & 322.3 \\
\hline neos-791021 & 51.3 \\
\hline neos-801834 & 6.6 \\
\hline neos- 808214 & 0.0 \\
\hline neos- 810286 & 8.3 \\
\hline neos- 810326 & 20.0 \\
\hline neos- 820879 & 14.1 \\
\hline neos- 824695 & 2.3 \\
\hline neos- 825075 & 22.4 \\
\hline neos- 826250 & 0.7 \\
\hline neos- 826812 & 0.5 \\
\hline neos- 827015 & 90.9 \\
\hline neos- 827175 & 0.0 \\
\hline neos- 829552 & 97.7 \\
\hline neos- 841664 & 18.4 \\
\hline s-847302 & 15.0 \\
\hline neos- 860300 & 36.3 \\
\hline neos- 885524 & \\
\hline neos- 892255 & 14.3 \\
\hline neos-906865 & 0.0 \\
\hline neos-932816 & 0.0 \\
\hline neos- 933364 & 6.9 \\
\hline neos-933562 & 21.7 \\
\hline neos-933815 & 7.3 \\
\hline neos-933966 & 226.7 \\
\hline neos- 934184 & 6.5 \\
\hline neos- 934278 & 72.2 \\
\hline
\end{tabular}

Copyright (C) by SIAM. Unauthorized reproduction of this article is prohibited. 
TABLE B.1

(Continued).

\begin{tabular}{|c|c|c|c|c|c|c|c|c|c|c|c|c|c|}
\hline \multirow[t]{3}{*}{ Name } & \multicolumn{4}{|c|}{ Gap } & \multicolumn{2}{|c|}{ Iterations } & \multicolumn{2}{|c|}{ MR } & Cuts & \multicolumn{2}{|c|}{ Time } & \multicolumn{2}{|c|}{ Success } \\
\hline & $\mathrm{Av}$ & age & & & & & & & & & & & \\
\hline & FP2.0 & $\mathrm{FPC}$ & FP2.0 & $\mathrm{FPC}$ & FP2.0 & FPC & FP2.0 & FPC & FPC & FP2.0 & FPC & FP2.0 & FPC \\
\hline neos-934441 & 54.2 & 58.4 & 24.1 & 31.2 & 7.6 & 4.4 & 0.0 & 0.0 & 0.0 & 10.4 & 9.5 & 10 & 10 \\
\hline neos-935234 & 42.6 & 52.7 & 20.0 & 22.9 & 4.9 & 5.4 & 0.0 & 0.0 & 0.0 & 10.0 & 10.3 & 10 & 10 \\
\hline neos-935348 & 37.3 & 55.1 & 21.1 & 17.3 & 4.5 & 4.0 & 0.0 & 0.0 & 1.5 & 9.3 & 8.9 & 10 & 10 \\
\hline neos-935674 & 2895.0 & 2630.0 & 1733.3 & 850.0 & 8.1 & 6.5 & 0.0 & 0.0 & 1.0 & 1.0 & 1.1 & 10 & 10 \\
\hline neos-935769 & 5.5 & 4.4 & 2.4 & 0.8 & 7.4 & 6.8 & 0.0 & 0.0 & 6.9 & 17.9 & 18.8 & 10 & 10 \\
\hline neos-941698 & 170.0 & 200.0 & 0.0 & 0.0 & 21.5 & 17.2 & 0.0 & 0.0 & 2.0 & 0.7 & 0.8 & 10 & 10 \\
\hline neos- 941782 & 20.0 & 25.0 & 5.6 & 0.0 & 18.9 & 19.6 & 0.0 & 0.0 & 8.8 & 1.0 & 1.5 & 10 & 10 \\
\hline neos- 942323 & 28.2 & 27.1 & 17.6 & 17.6 & 19.9 & 25.0 & 0.0 & 0.0 & 6.0 & 0.3 & 0.6 & 10 & 10 \\
\hline neos-948268 & 0.0 & 0.0 & 0.0 & 0.0 & 3.5 & 3.6 & 0.0 & 0.0 & 0.0 & 10.6 & 10.7 & 10 & 10 \\
\hline neos-955215 & 2.2 & 1.7 & 0.4 & 0.4 & 4.3 & 4.6 & 0.0 & 0.0 & 2.4 & 0.0 & 0.3 & 10 & 10 \\
\hline 957323 & 0.2 & 0.3 & 0.0 & 0.0 & 5.3 & 4.6 & 0.0 & 0.0 & 0.5 & 5.7 & 19.1 & 10 & 10 \\
\hline neos- 1067731 & 0.6 & 5.4 & 0.4 & 0.4 & 5.1 & 5.8 & 0.0 & 0.0 & 0.3 & 2.4 & 4.4 & 10 & 10 \\
\hline neos- 1109824 & 55.4 & 43.4 & 37.8 & 8.2 & 24.7 & 18.4 & 0.7 & 0.2 & 45.0 & 0.7 & 1.8 & 10 & 10 \\
\hline neos- 1171448 & 1.1 & 0.6 & 0.3 & 0.0 & 14.8 & 13.2 & 0.0 & 0.0 & 20.5 & 2.0 & 18.2 & 10 & 10 \\
\hline neos- 1200887 & 12.4 & 8.9 & 5.4 & 2.7 & 11.4 & 13.6 & 0.0 & 0.0 & 12.8 & 0.1 & 1.0 & 10 & 10 \\
\hline neos-1211578 & 4.5 & 2.8 & 2.6 & 1.3 & 13.6 & 10.9 & 0.0 & 0.0 & 12.2 & 0.0 & 0.7 & 10 & 10 \\
\hline
\end{tabular}

TABLE B.2

Detailed statistics for FP2.0 and FPC for problems in class B.

\begin{tabular}{|c|c|c|c|c|c|c|c|c|c|c|c|c|c|}
\hline \multirow[t]{3}{*}{ Name } & \multicolumn{4}{|c|}{ Gap } & \multicolumn{2}{|c|}{ Iterations } & \multicolumn{2}{|c|}{$\mathrm{MR}$} & \multirow[t]{2}{*}{ Cuts } & \multicolumn{2}{|c|}{ Time } & \multicolumn{2}{|c|}{ Success } \\
\hline & Ave & age & $\bar{B}$ & & & & & & & & & & \\
\hline & FP2.0 & $\mathrm{FPC}$ & FP2.0 & FPC & FP2.0 & FPC & FP2.0 & FPC & FPC & FP2.0 & $\mathrm{FPC}$ & FP2.0 & FPC \\
\hline 10teams & 10.9 & 9.7 & 4.5 & 3.2 & 109.0 & 89.3 & 2.0 & 0.0 & $\begin{array}{ll}6.7 \\
\end{array}$ & 7.3 & 6.6 & 10 & 10 \\
\hline dano3mip & 12.3 & 7.9 & 6.9 & 6.1 & 68.5 & 85.9 & 0.4 & 0.1 & 994.9 & 330.2 & 1536.6 & 10 & 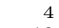 \\
\hline re 1 & 560.0 & 27120.0 & 21900.0 & 12200.0 & 68.4 & 67.9 & 3.4 & 0.7 & 68.1 & 0.1 & 2.8 & 10 & 10 \\
\hline markshare2 & 59110.0 & 39830.0 & 26700.0 & 25600.0 & 70.0 & 69.7 & 3.0 & 0.3 & 64.4 & 0.2 & 3.1 & 10 & 10 \\
\hline opt1217 & 3.7 & 0.0 & 0.0 & 0.0 & 38.3 & 33.7 & 0.6 & 0.1 & 7.1 & 0.1 & 1.3 & 10 & 10 \\
\hline pk1 & 574.5 & 115.1 & 272.7 & 63.6 & 57.6 & 278.8 & 1.4 & 0.6 & 294.3 & 0.2 & 13.1 & 10 & 9 \\
\hline swath & 164.1 & 130.6 & 65.2 & 35.6 & 162.5 & 370.5 & 5.0 & 0.1 & 606.2 & 10.1 & 231.5 & 10 & 6 \\
\hline $\operatorname{tr} 12-30$ & 27.5 & 37.2 & 23.5 & 22.6 & 27.0 & 21.4 & 0.3 & 0.0 & 835.6 & 0.1 & 3.3 & 10 & 10 \\
\hline vpm2 & 27.3 & 19.5 & 12.7 & 36 & 17.0 & 11.6 & 0.4 & $0 . \mathrm{r}$ & 26.2 & 0.0 & 0.5 & 10 & 10 \\
\hline 22433 & 0.3 & 0.1 & 0.0 & 0.0 & 65.0 & 67.8 & 6.1 & 0.2 & 18.7 & 0.3 & 1.4 & 10 & 10 \\
\hline 23588 & 2.4 & 1.9 & 1.1 & 1.0 & 91.8 & 71.7 & 4.3 & 0.1 & 7.7 & 0.3 & 0.8 & 10 & 10 \\
\hline bienst1 & 13.9 & 13.0 & 0.0 & 0.0 & 53.5 & 102.8 & 2.7 & 0.5 & 537.2 & 0.5 & 34.6 & 10 & 10 \\
\hline bienst2 & 15.0 & 7.5 & 9.3 & 2.1 & 54.7 & 79.4 & 2.5 & 0.6 & 578.7 & 0.6 & 24.9 & 10 & 10 \\
\hline mcf2 & 19.3 & 22.8 & 1.1 & 9.6 & 96.2 & 455.4 & 20.4 & 0.3 & 2403.2 & 2.7 & 449.7 & 10 & 7 \\
\hline 522351 & 36.8 & 2.3 & 19.2 & 0.0 & 50.4 & 27.1 & 1.8 & 1.7 & 263.8 & 0.4 & 3.0 & 10 & 10 \\
\hline 582605 & 0.0 & 0.0 & 0.0 & 0.0 & 67.5 & 45.5 & 2.6 & 0.4 & 80.5 & 0.8 & 8.8 & 10 & 10 \\
\hline 583731 & 0.0 & 0.0 & 0.0 & 0 . & 37.9 & 43.5 & 0.1 & $0 .($ & 190.6 & 0.2 & 1.4 & 10 & 10 \\
\hline 631164 & 11.6 & 11.9 & 7.0 & & 75.8 & 70.0 & 1.2 & 0.2 & 64.5 & 0.4 & 1. & 10 & 10 \\
\hline 631517 & 10.3 & 10.1 & 7.5 & 8 & 75.4 & 72.7 & 0.9 & 0.4 & 53.5 & 0.3 & 0. & 10 & 10 \\
\hline 777800 & 0.0 & 0.0 & 0.0 & & & 32.6 & .7 & & 2.6 & 9.3 & 16 & 10 & 10 \\
\hline 831188 & 12.4 & 15.5 & 1.8 & 1 & 137.8 & 176.5 & 4.4 & 0.0 & 30.7 & 11.9 & 21.7 & 10 & 10 \\
\hline 9838 & 33.9 & 22.4 & 12.9 & & 56.9 & 234.4 & .7 & 15.0 & 124.9 & 3.6 & 51. & 10 & 8 \\
\hline 9859 & 235.9 & 39.7 & & & 42.2 & 15 & & 0 & 30.9 & 0.5 & & 10 & 10 \\
\hline 886822 & 44.9 & 38.7 & 24.2 & 22 & 114.0 & 112.2 & 3 & 0. & 27.2 & 2.0 & 5 & 10 & 10 \\
\hline 911880 & 210.1 & 51.8 & 152.3 & 11.2 & 51.0 & 36.7 & 1.0 & 0.6 & 101.1 & 0.1 & 2.0 & 10 & 10 \\
\hline 1970 & 230.0 & 44.7 & 115.0 & & 50.2 & & & & 126.0 & 0 & & 10 & 10 \\
\hline 2010 & 52.9 & 55.0 & 28.6 & 21 & 164.9 & 188.5 & 2.6 & 0. & 7.7 & 8.7 & 11. & 10 & 10 \\
\hline 053234 & 111.8 & 51.0 & 68.2 & 27.6 & $\begin{array}{r}39.1 \\
\end{array}$ & 34.6 & 4.5 & 0.8 & 112.7 & 0.4 & 6.7 & 10 & 10 \\
\hline 20495 & 56.3 & 50.8 & 21.3 & 18.6 & 17.3 & 14.7 & 0. & 0. & 35.1 & 0. & 1. & 10 & 10 \\
\hline neos-1121679 & 2799.4 & 1681.3 & 1506.3 & 700.0 & 68.3 & 69.7 & 2.2 & 0.5 & 74.9 & 0.1 & 2.9 & 10 & 10 \\
\hline
\end{tabular}

TABLE B.3

Detailed statistics for FP2.0 and FPC for problems in class $C$.

\begin{tabular}{|c|c|c|c|c|c|c|c|c|c|c|c|c|c|}
\hline \multirow{2}{*}{ Name } & \multicolumn{4}{|c|}{ Gap } & \multicolumn{2}{|c|}{ Iterations } & \multicolumn{2}{|c|}{$\mathrm{MR}$} & \multirow[t]{2}{*}{$\begin{array}{l}\text { Cuts } \\
\end{array}$} & \multicolumn{2}{|c|}{ Time } & \multicolumn{2}{|c|}{ Success } \\
\hline & $\begin{array}{r}\text { Ave } \\
\mathrm{FP} 2.0 \\
\end{array}$ & & $\mathrm{FP} 2.0$ & & FP: & & & & & & & & \\
\hline a1c1s1 & 60.1 & 34.9 & 38.6 & 21.8 & 51.3 & 18.6 & & 0.0 & 744.0 & & 3.7 & & $\overline{10}$ \\
\hline noint & 17.8 & $\begin{array}{l}18.1 \\
18.1\end{array}$ & 5.1 & 10.4 & 112.3 & 353.5 & & 0.3 & 2039.5 & 2.6 & 185.0 & 10 & \\
\hline ass 4 & 294.6 & 141.9 & 138.9 & & 218.3 & 119.1 & 101.9 & 4.1 & 1455.1 & 0.3 & & 10 & 10 \\
\hline & 203.0 & 142.7 & 169.6 & 93.0 & 152.2 & 120.1 & 12.9 & 2.1 & 3857.0 & 1.9 & 53.0 & 10 & 10 \\
\hline mas74 & 72.3 & 17.7 & 43.1 & 9.1 & 6.2 & & .9 & 0.4 & 528.6 & 0.1 & 44.3 & 7 & 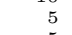 \\
\hline $\operatorname{mas} 76$ & & & & & 106.2 & 481.9 & 16.7 & 0.3 & 365.0 & 0.2 & 25.9 & 7 & 5 \\
\hline modglob & $3 \times 10^{6}$ & $4 \times 10^{6}$ & $6.5 \times 10^{6}$ & $\times 10^{6}$ & 66.1 & & 27.2 & 1.5 & 81.5 & 0.1 & 1.7 & 10 & \\
\hline & & & & & & & & & & 0.1 & & 10 & 10 \\
\hline align & 1.3 & 1.4 & 0.9 & & 249.4 & & & 0.0 & & 9.1 & 12.6 & 9 & 9 \\
\hline $\mathrm{ne}$ & & 203.3 & & 197.7 & 843.7 & & 12 & 1.1 & 1304.7 & 9.5 & 438.0 & 0 & \\
\hline $\begin{array}{l}\text { neos4 } \\
\text { neos17 }\end{array}$ & 65.5 & & 516 & $\begin{array}{r}5.1 \\
23.0\end{array}$ & $\begin{array}{l}614.6 \\
689.5\end{array}$ & $\begin{array}{ll}119.7 \\
144.8\end{array}$ & $\begin{array}{l}285.9 \\
659.1\end{array}$ & 0.2 & 31.6 & 40.7 & 79.5 & & 10 \\
\hline - 43 & $\begin{array}{r}53.5 \\
592.0\end{array}$ & $\begin{array}{r}56.5 \\
521.6\end{array}$ & $\begin{array}{r}51.6 \\
485.4\end{array}$ & $\begin{array}{r}23.0 \\
164.7\end{array}$ & \begin{tabular}{|l|l|}
699.5 \\
1208.6
\end{tabular} & & $\begin{array}{l}659.1 \\
266.4\end{array}$ & 32.5 & & $\begin{array}{l}1.6 \\
5.3\end{array}$ & $\begin{array}{l}18.8 \\
27.7\end{array}$ & & $\begin{array}{l}10 \\
10\end{array}$ \\
\hline & 159.0 & 25.3 & 103.4 & & & & & 0.8 & & .0 & 10.8 & 7 & 10 \\
\hline & 139.2 & 1.0 & 31.3 & & & & & & & 5 & & 10 & 10 \\
\hline & & & & & & & & & & & & 0 & 10 \\
\hline & & & 7.8 & .5 & & & & & & & 38.8 & 2 & 10 \\
\hline & 11.0 & & & 7 & & & & & & & & 10 & 10 \\
\hline & $\begin{array}{l}51.3 \\
7.3\end{array}$ & $\begin{array}{r}38.1 \\
9.1\end{array}$ & $\begin{array}{l}44.7 \\
2.7\end{array}$ & 8.7 & & 24 & $\begin{array}{l}168.1 \\
07.8\end{array}$ & 3.8 & $\begin{aligned} 223.9 \\
16.5\end{aligned}$ & 3 & 15.5 & 2 & 10 \\
\hline & 18.2 & 21 & 8.8 & & & & & $\begin{array}{r}0.8 \\
0.8\end{array}$ & & & & & 10 \\
\hline & 09350.2 & 7319 & 67875.2 & 526.8 & & & & 2.5 & & 117.3 & & to & 10 \\
\hline & 15.4 & 9 & 11. & & & & & & & & & 10 & 10 \\
\hline & & & & & 105 & & & & & & & 0 & 9 \\
\hline & $\begin{array}{r}46.2 \\
0.0\end{array}$ & $\begin{array}{r}32 \\
0\end{array}$ & $\begin{aligned} 15.4 \\
0.0\end{aligned}$ & & $\begin{array}{r}24 \\
105\end{array}$ & & & & .2 & & & 10 & 10 \\
\hline & $\begin{array}{r}0.0 \\
27.2\end{array}$ & 16 & $\begin{array}{r}0.0 \\
10.8\end{array}$ & & & & & & & & 14.3 & 3 & 10 \\
\hline & & & & & & & 12 & 3 & & $\begin{array}{r}2.8 \\
0.3\end{array}$ & & $\begin{array}{l}9 \\
0\end{array}$ & 10 \\
\hline & & & & & & & & & & & & 0 & 10 \\
\hline & & & & & & & & & 15 & & -8 & 10 & 10 \\
\hline 1173026 & 63.2 & 34.1 & 35.5 & 20. & 54.0 & 45.9 & 8. & 4. & 70.9 & 0.2 & 5.2 & 10 & \\
\hline
\end{tabular}

Copyright (c) by SIAM. Unauthorized reproduction of this article is prohibited. 
Acknowledgments. The authors would like to thank Prof. Matteo Fischetti and Dr. Domenico Salvagnin for kindly providing the original code for FP 2.0 [18], which we modified in order to perform our numerical testing. Thanks also to Matteo Fischetti for many stimulating discussions on the FP and related algorithmic issues.

\section{REFERENCES}

[1] T. Achterberg and T. Berthold, Improving the feasibility pump, Discrete Optim., 4 (2007), pp. $77-86$.

[2] E. Balas, S. Ceria, M. Dawande, F. Margot, and G. Pataki, octane: A new heuristic for pure 0-1 programs, Oper. Res., 49 (2001), pp. 207-225.

[3] E. Balas and C.H. Martin, Pivot and complement-a heuristic for 0-1 programming, Management Sci., 26 (1980), pp. 86-96.

[4] E. Balas, S. Schmieta, AND C. Wallace, Pivot and shift-a mixed integer programming heuristic, Discrete Optim., 1 (2004), pp. 3-12.

[5] L. Bertacco, M. Fischetti, and A. Lodi, A feasibility pump heuristic for mixed-integer problems, Discrete Optim., 4 (2007), pp. 63-76.

[6] T. Berthold, Primal Heuristics for Mixed Integer Programs, MS thesis, Technische Universität Berlin, 2006.

[7] D.P. Bertsekas, Nonlinear Programming, 2nd ed., Athena Scientific, Boston, 1999.

[8] P. Bonami, G. Cornuejols, A. Lodi, and F. Margot, Feasibility pump for mixed integer nonlinear programs, Math. Program., 119 (2009), pp. 331-352.

[9] R.S. Burachik And A.N. Iusem, Set-Valued Mappings and Enlargements of Monotone Operators, Optim. Appl. 8, Springer, New York, 2008.

[10] C. D'Ambrosio, A. Frangioni, L. Liberti, And A. Lodi, Experiments with a feasibility pump approach for nonconvex MINLPs, in Symposium on Experimental Algorithms, P. Festa, ed., Lecture Notes in Comput. Sci. 6049, Springer, New York, 2010, pp. 350-360.

[11] C. D'Ambrosio, A. Frangioni, L. Liberti, and A. Lodi, A Storm of Feasibility Pumps for Nonconvex MINLP, http://www.di.unipi.it/ frangio/abstracts.html.

[12] A. Danillidis and C. Lemarechal, On a primal-proximal heuristic in discrete optimization, Math. Program., 104 (2005), pp. 105-128.

[13] E. Danna, E. Rothberg, and C.L. Pape, Exploring relaxation induced neighborhoods to improve MIP solutions, Math. Program., 102 (2005), pp. 71-90.

[14] J. Eckstein And M. Nediak, Pivot, cut and dive: A heuristic for 0-1 mixed integer programming, J. Heuristics, 13 (2007), pp. 471-503.

[15] B.H. FAAland AND F.S. HillieR, Interior path methods for heuristic integer programming procedures, Oper. Res., 27 (1979), pp. 1069-1087.

[16] M. Fischetti And A. Lodi, Local branching, Math. Program., 98 (2003), pp. 23-47.

[17] M. Fischetti, F. Glover, And A. Lodi, The feasibility pump, Math. Program., 104 (2005), pp. $91-104$

[18] M. Fischetti and D. Salvagnin, Feasibility Pump 2.0, Math. Program. Comput., 1 (2009), pp. 201-222.

[19] S. Ghosh and R. Hayward, Pivot and Gomory Cut: A 0-1 Mixed Integer Programming

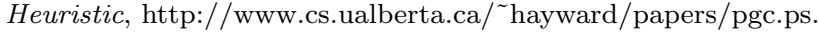

[20] F.S. HiLlieR, Efficient heuristic procedures for integer linear programming with an interior, Oper. Res., 17 (1969), pp. 600-637.

[21] T. Ibaraki, T. Ohashi, and H. Mine, A heuristic algorithm for mixed-integer programming problems, Math. Program. Stud., 2 (1974), pp. 115-136.

[22] N. Karmarkar, M.G.C. Resende, and K.G. Ramakrishnan, An interior point algorithm to solve computationally difficult set covering problems, Math. Program., 52 (1991), pp. 597618.

[23] A. Lokketangen And F. Glover, Solving zero-one mixed integer programming problems using tabu search, European J. Oper. Res., 106 (1998), pp. 624-658.

[24] S. LuCidi AND F. RinAldi, Exact penalty functions for nonlinear integer programming problems, J. Optim. Theory Appl., 145 (2010), pp. 479-488.

[25] R.T. RockAfEllar, Monotone operators and the proximal point algorithm, SIAM J. Control Optim., 14 (1976), pp. 877-898.

[26] M. De Santis, S. Lucidi, And F. Rinaldi, New Concave Functions for Improving the Feasibility Pump, http://www.optimization-online.org/DB_HTML/2010/07/2667.html.

Copyright (c) by SIAM. Unauthorized reproduction of this article is prohibited. 